\title{
Parkinson Disease: from Pathophysiology to the Animal Models
}

\author{
Somayeh Vazifehkhah ${ }^{1,2}$, Fariba Karimzadeh ${ }^{3 *}$ \\ ${ }^{1}$ Shefa Neuroscience Research Center, Khatam Alanbia Hospital, Tehran, Iran \\ ${ }^{2}$ Department of Physiology, Iran University of Medical Sciences, Tehran, Iran \\ ${ }^{3}$ Cellular and Molecular Research Center, Iran University of Medical Sciences, Tehran, Iran
}

Received: 5 Apr 2016

\section{Article Info:}

Accepted: 5 Aug 2016

\section{ABSTRACT}

Introduction: Parkinson disease (PD) is a severe and progressive neurodegenerative disorder in the central nervous system. The most features of this disease are several parts-substantia nigra compacta cell-loss, and accumulation of aggregated $\alpha$-synuclein in specific brain stem, spinal cord, and cortical regions. The main risk factors are age and environmental factors. Several genes have been identified for inheritance PD. Identification of these genes had been leaded to provide new solutions. Dopamine replacement therapy and commonly used drugs significantly reduce motor handicaps and had a positive effect on the quality of life improvement. Animal models are important in investigation of the mechanisms involved in the pathogenesis of PD and therapeutic strategies. Conclusion: Despite numerous models to induce PD, MPTP and 6-OHDA models have been more frequently used. In this study, the most important factors involved in the pathophysiology of PD as well as the most current animal models, are described.

*Corresponding Author: Fariba Karimzadeh

E-mail: Fariba_karimzade@yahoo.com 


\title{
بيمارى ياركينسون: از ياتوفيزيولوزى تا مدلهاى حيوانى
}

\author{
سميه وظيفه خواه ‘'، فريبا كريم زاده"r \\ 'مركز تحقيقات علوم اعصاب شفا، بيمارستان خاتمالانبياء، تهران، ايران \\ كَّروه فيزيولوزى، دانشعاه علوم يزشكى ايران، تهران، ايران

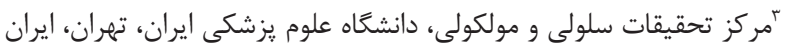

كليد وازهها:

l. بيمارى : ياركينسون

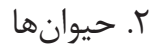

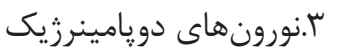

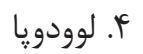

مقدمه: بيمارى يار كينسون يك اختلال تحليل برنده عصبى ييشرونده و شديد در سيستم اعصاب مركزى

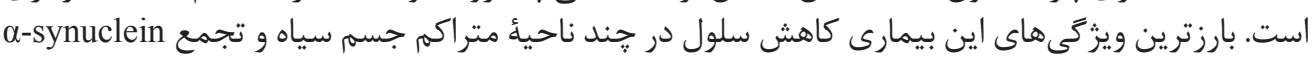

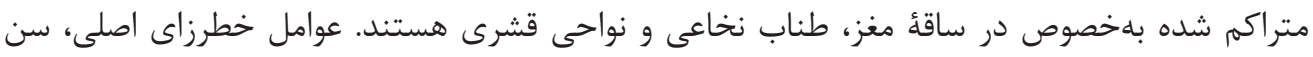

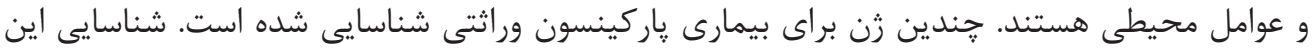

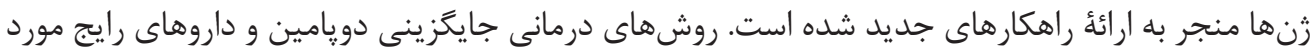

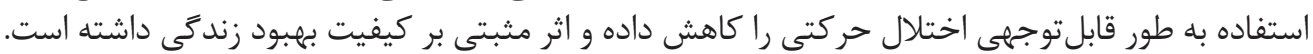

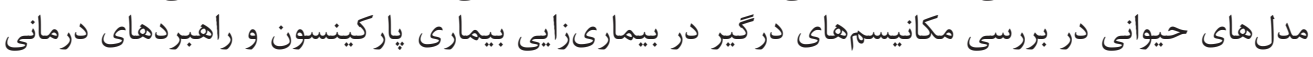

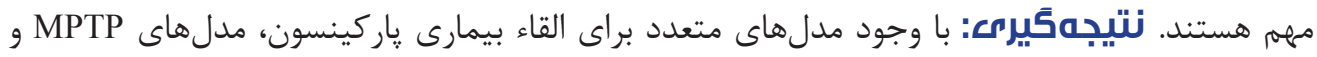

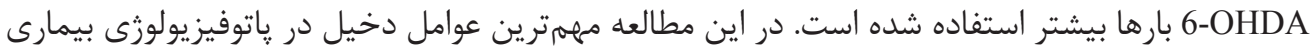

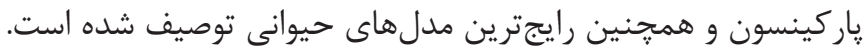

" نويسنده مسئول: فريبا كريم زاده

آدرس الكترونيكى: Fariba_karimzade@yahoo.com 


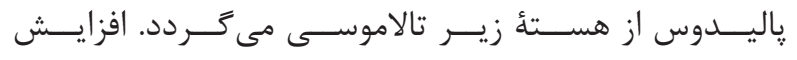

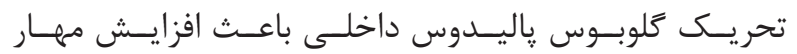

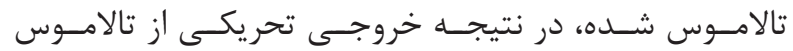

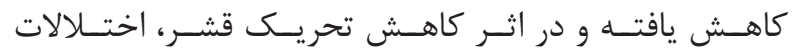

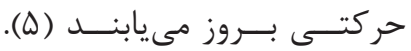
علايم زيستى بيمارى هار كينسون

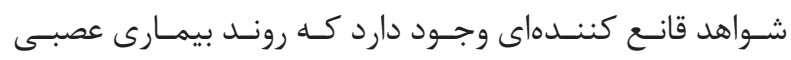

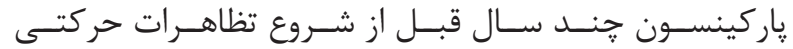

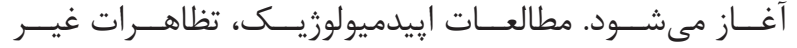

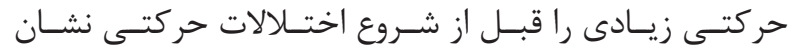

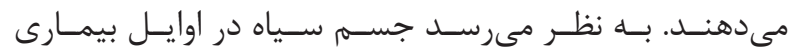

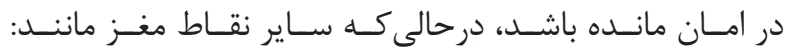

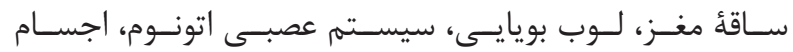

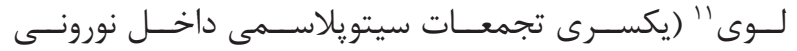

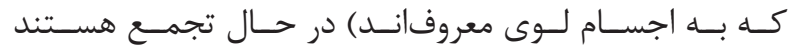

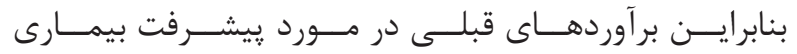

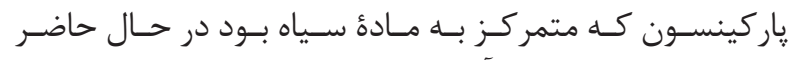

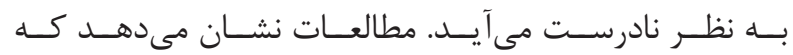

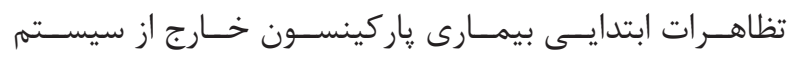

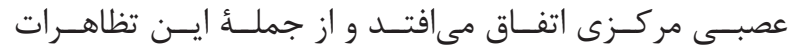

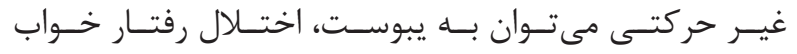

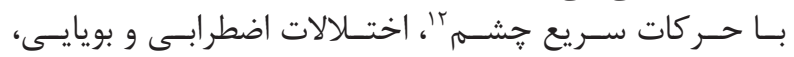

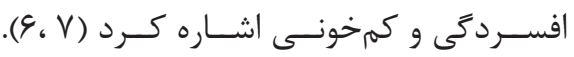

\section{عوامل دخيل در بيمارى هار كينسون}

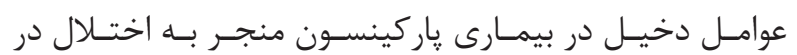

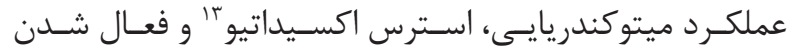

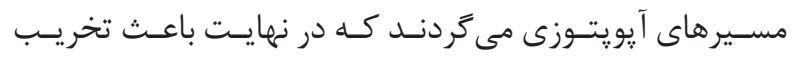

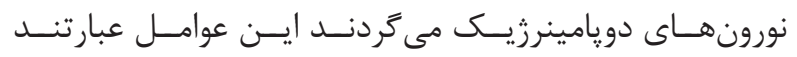

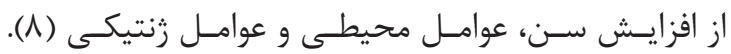

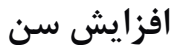

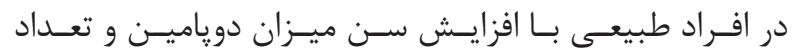

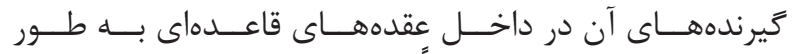

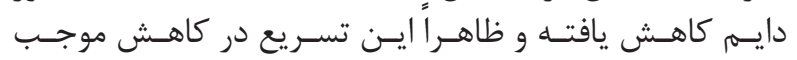

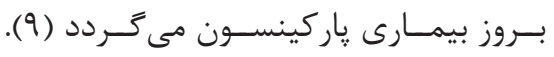

\section{عوامل محيطى}

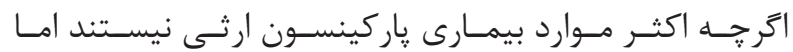

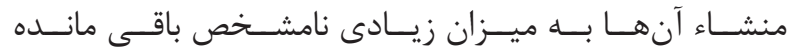

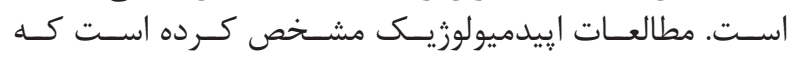

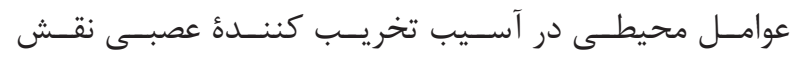

${ }^{1}$ Parkinson disease

${ }^{2}$ Akinesis

${ }^{3}$ Bradykinesia

${ }^{4}$ Locus coeruleus

${ }^{5}$ Raphe nucleus

${ }^{6}$ Meynert nucleus

${ }^{7}$ Dorsal motor nucleus of vagus

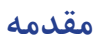

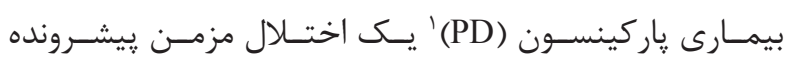

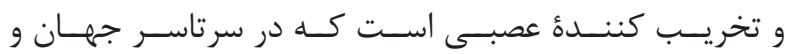

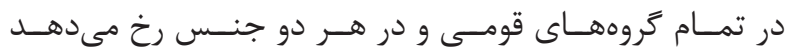

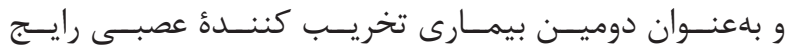

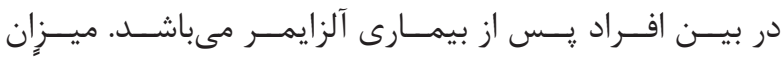

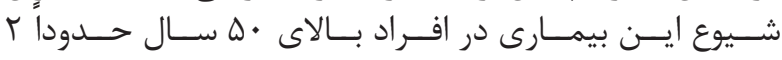

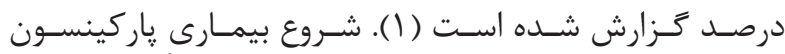

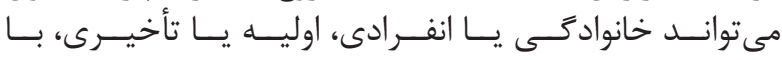

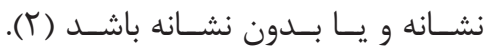

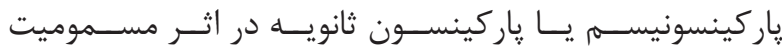

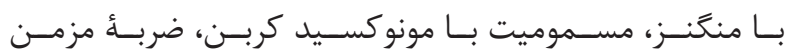

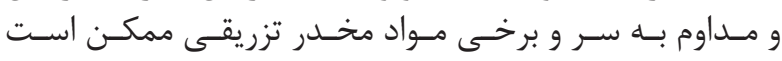

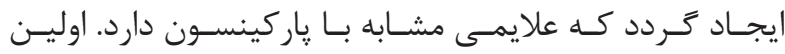

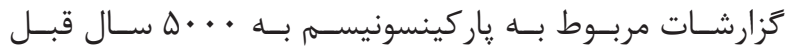

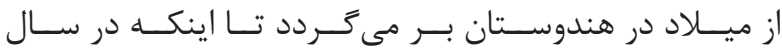
IN IV

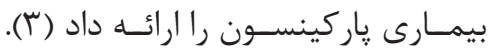

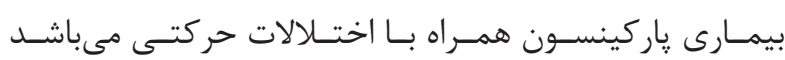

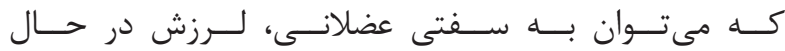

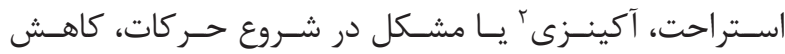

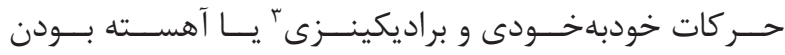

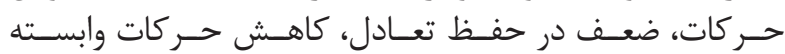

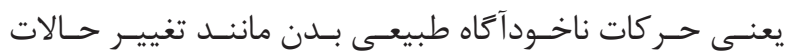

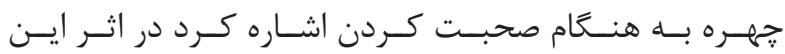

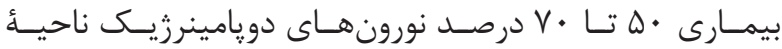

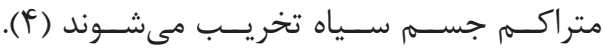

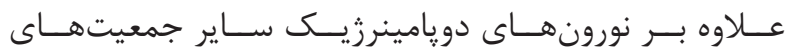

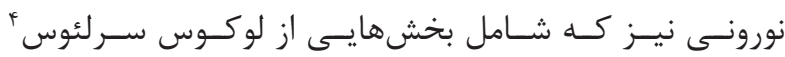

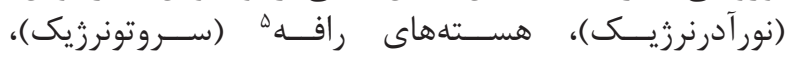

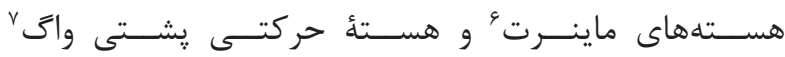

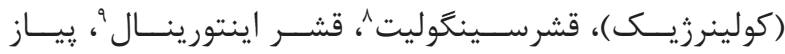

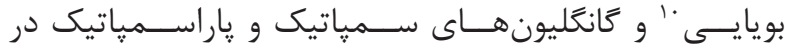

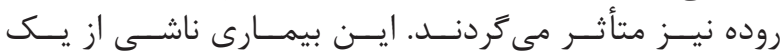

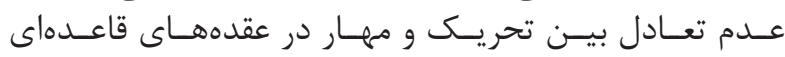

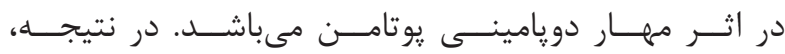

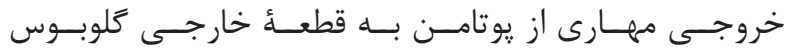

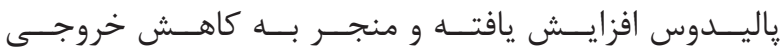

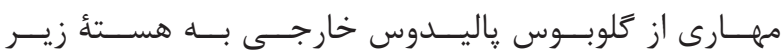

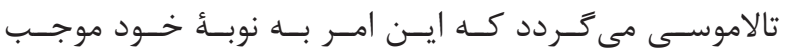

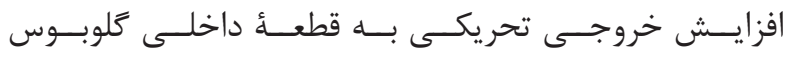

\footnotetext{
${ }^{8}$ Cingulate cortex

${ }^{9}$ Entorinal cortex

${ }^{10}$ Olfactory bulb

${ }^{11}$ Lewy body

${ }^{12}$ Rapid eye movement sleep behavior disorder

${ }^{13}$ Oxidative stress
} 


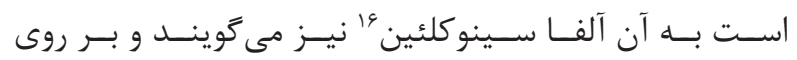

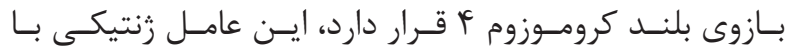

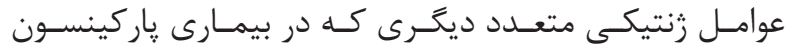

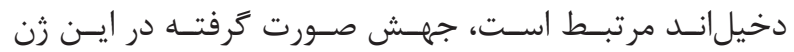

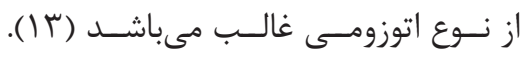

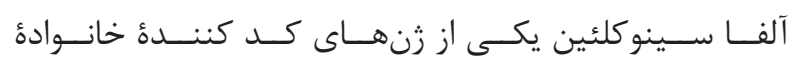

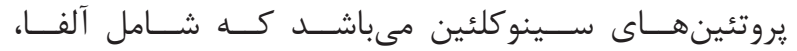

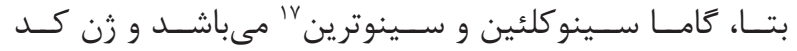

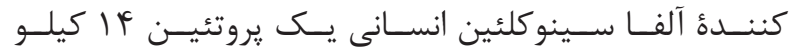

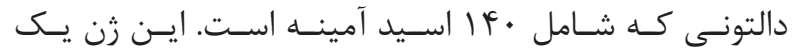

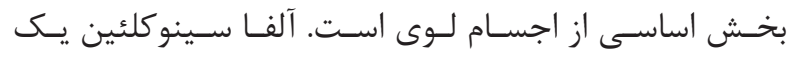

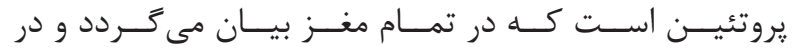

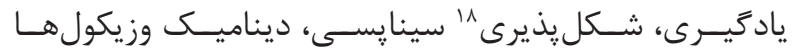

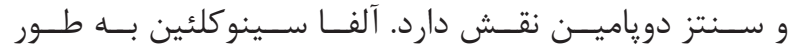

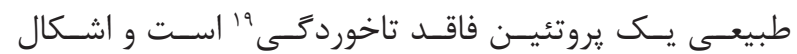

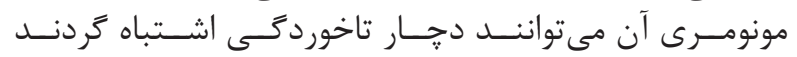

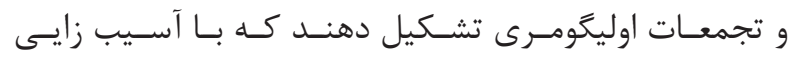

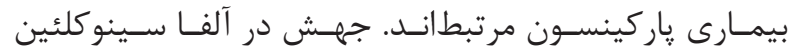

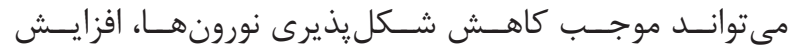

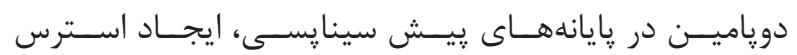

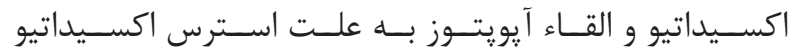

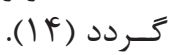

PARK2

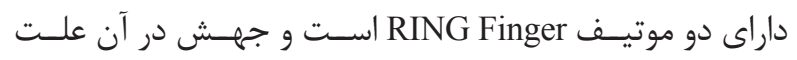

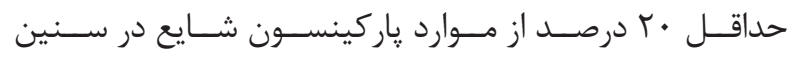

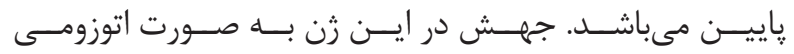

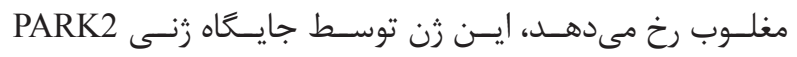

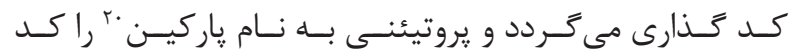

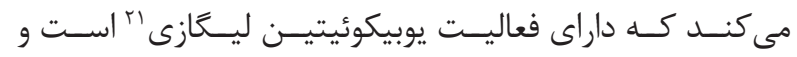

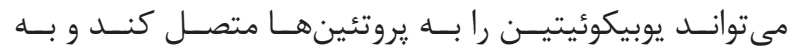

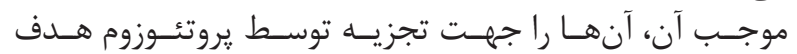

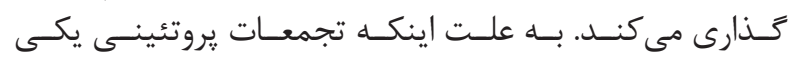

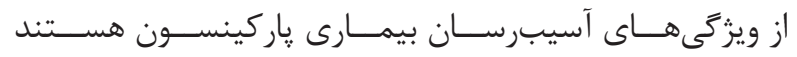

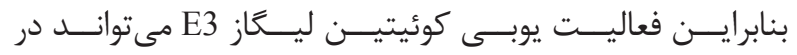

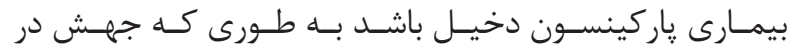

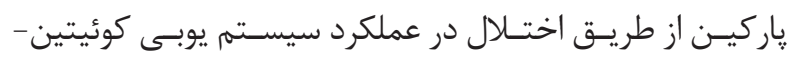

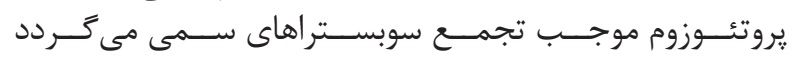

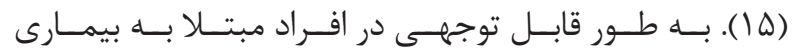

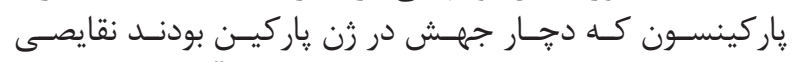

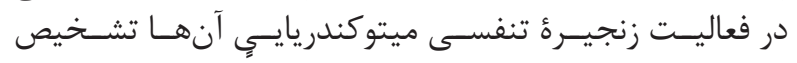

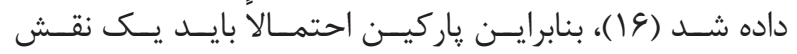

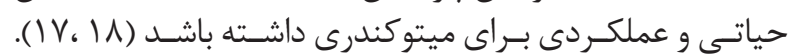

PARK3

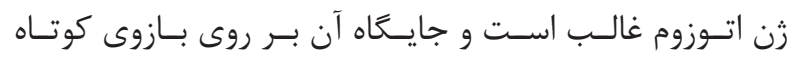

${ }^{14} 1$-Methyl-4-phenyl-1,2,3,6-tetrahydropyridine

${ }^{15}$ Reactive oxygen spices

${ }^{16} \alpha$-Synuclien

${ }^{17}$ Synoterin

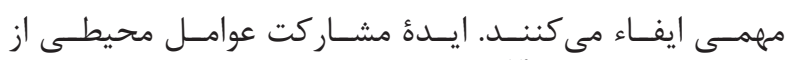

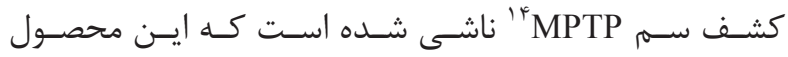

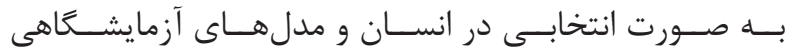

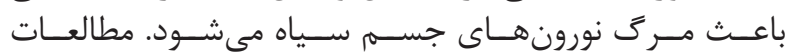

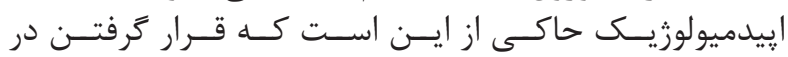

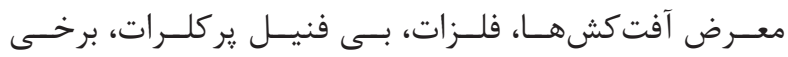

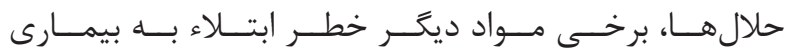

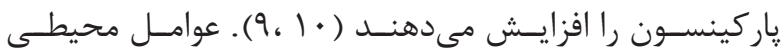

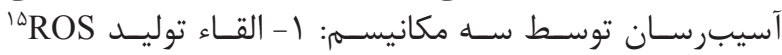

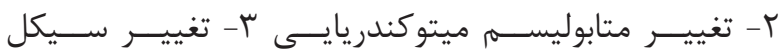

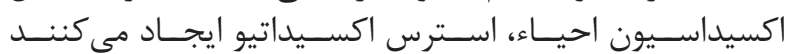

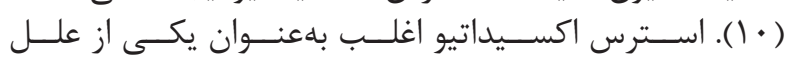

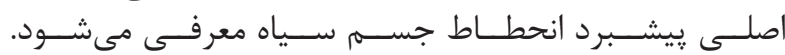

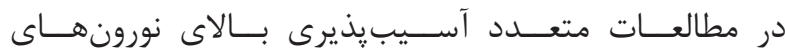

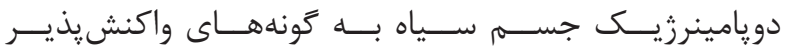

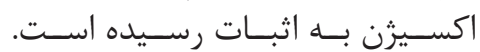

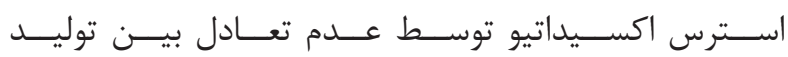

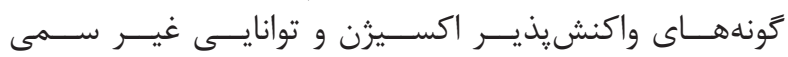

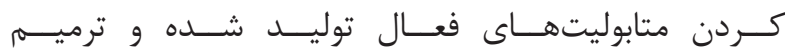

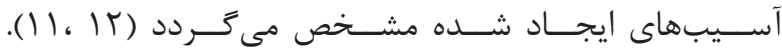

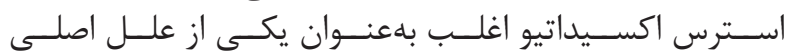

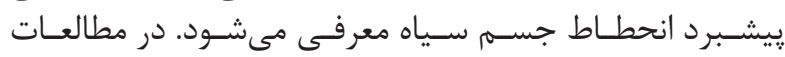

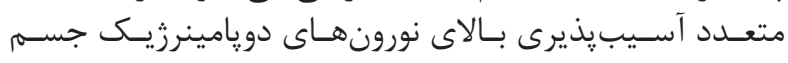

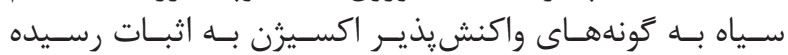

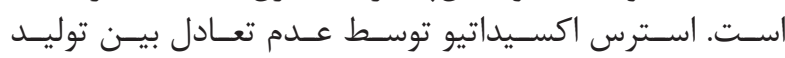

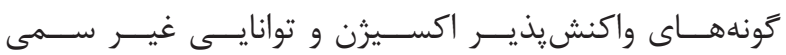

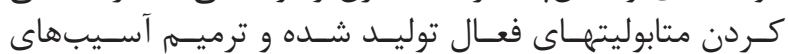

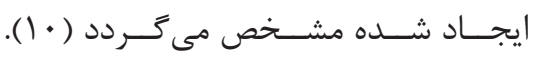

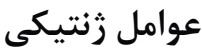

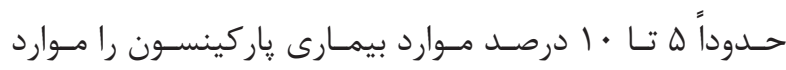

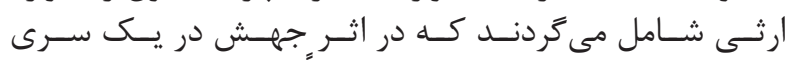

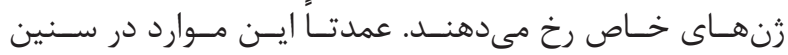

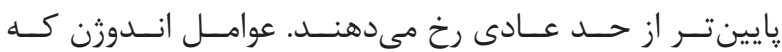

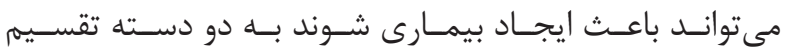

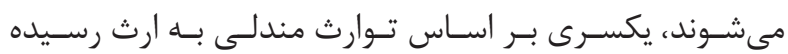

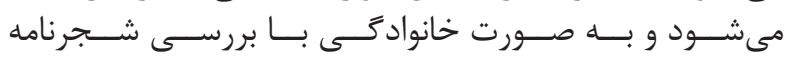

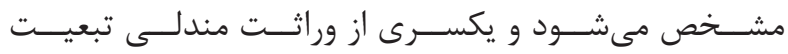

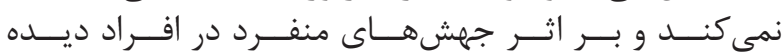

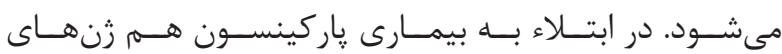

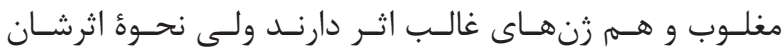

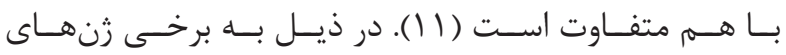

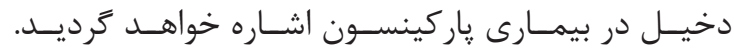

\section{PARK1}

اوليـن زن مرتبــط بــا اشـكال ارثـى بيمــارى بار كينســون

${ }^{18}$ Plastisity

${ }^{19}$ Folding

${ }^{20}$ Parkin

${ }^{21}$ Ubiqutin ligas 


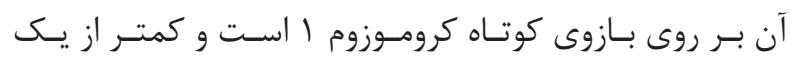

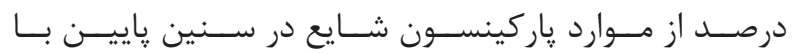

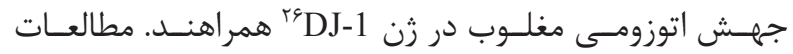

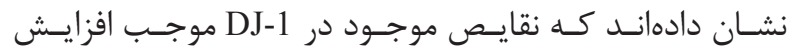

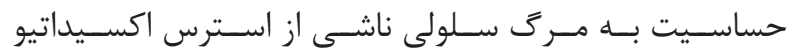

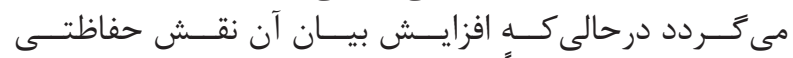

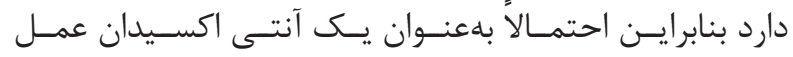

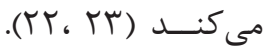

PARK8

از و و همكارانسش، Funayama

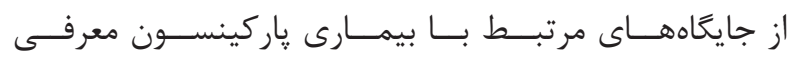

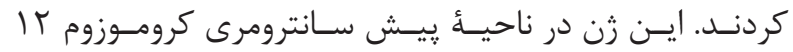

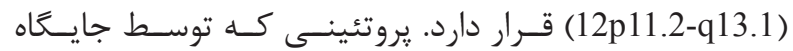

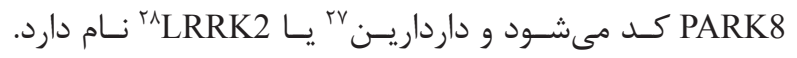
LRRK2

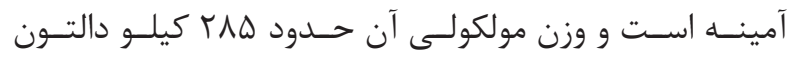

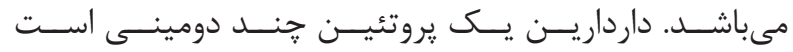

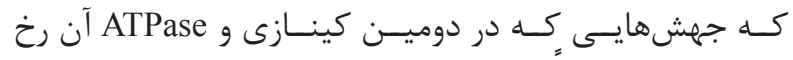

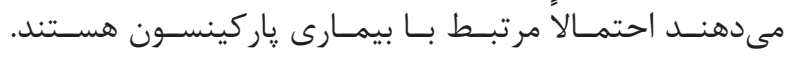

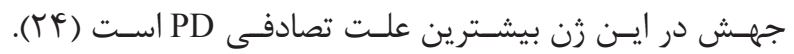

PARK9

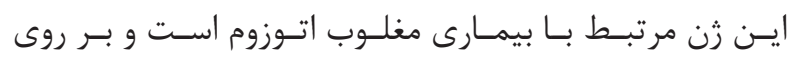

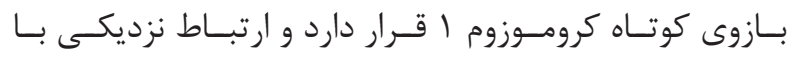

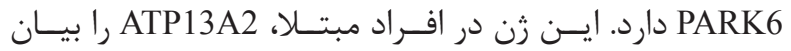

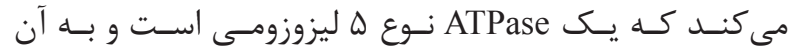

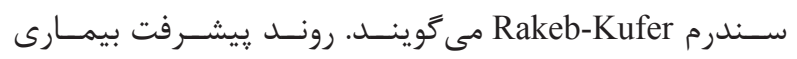

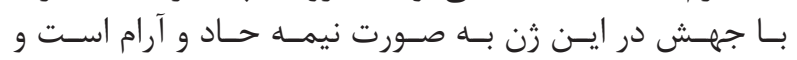

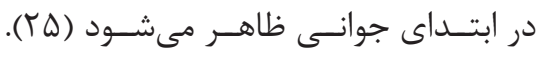

PARK10

جهـش در ايـن زن مرتبـط بــا بيمـارى غالـب اتـوزوم اسـت و و

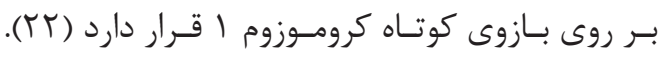

\section{PARK11}

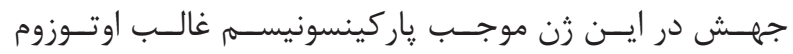

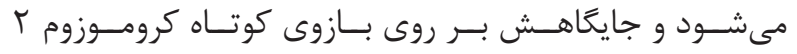

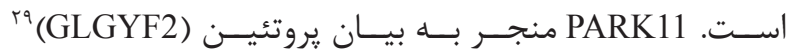

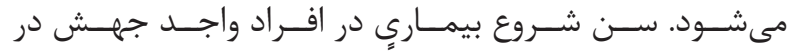

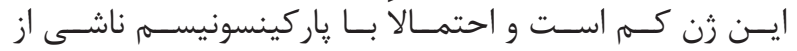

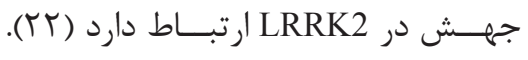

PARK12

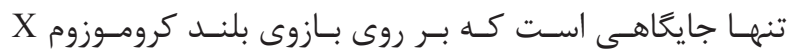

${ }^{22}$ Teremor

${ }^{23}$ Ubiquitin carboxy terminal hydrolase L1

${ }^{24}$ Deubiquin

${ }^{25}$ PTEN-induced kinase 1

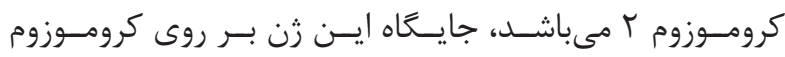

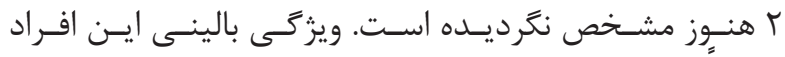

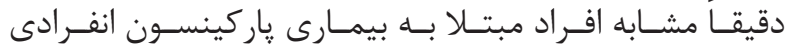

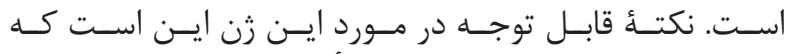

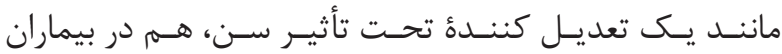

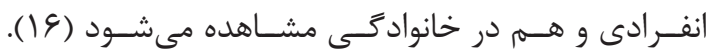

PARK4

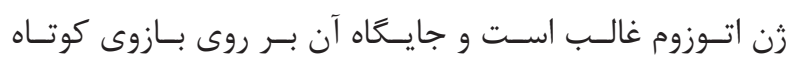

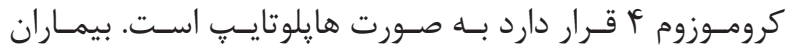

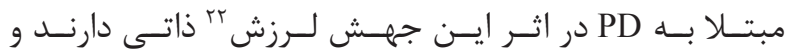

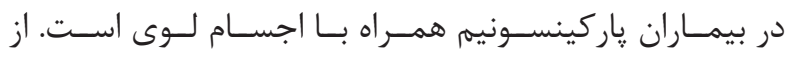

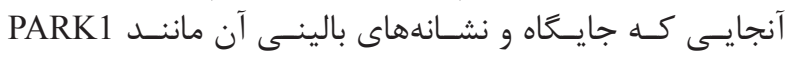

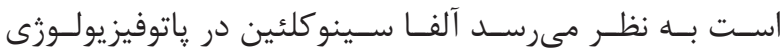

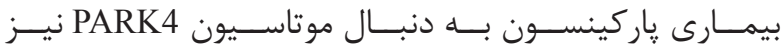

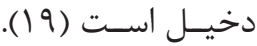

PARK5

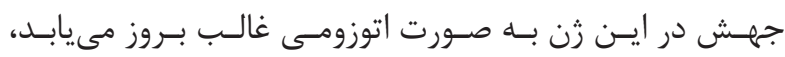

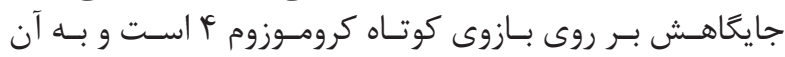
كوئيسن

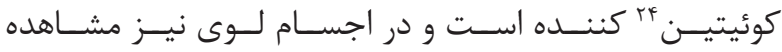

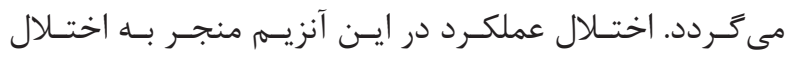

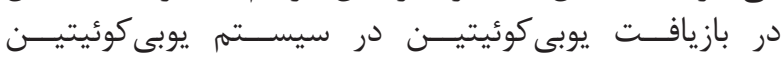

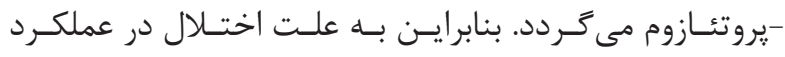

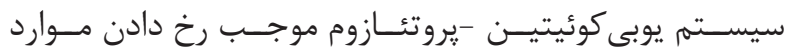

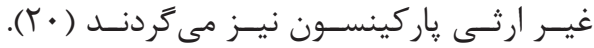

PARK6

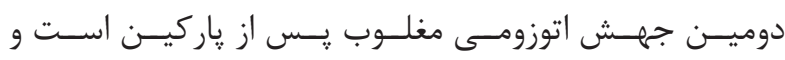

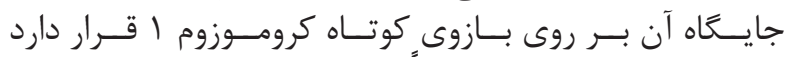

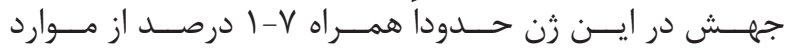

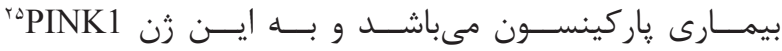

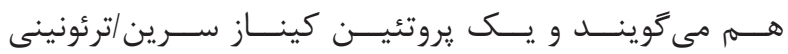

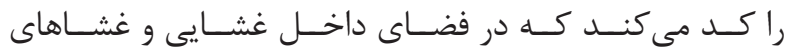

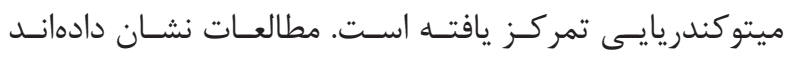

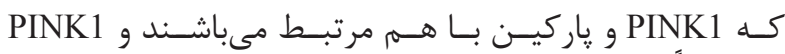

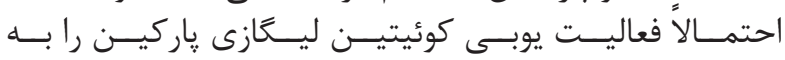

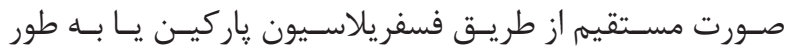

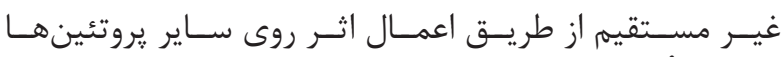

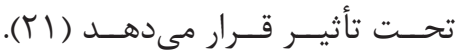

PARK7

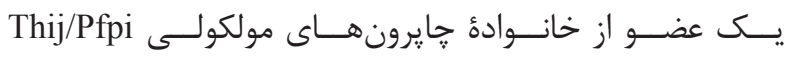

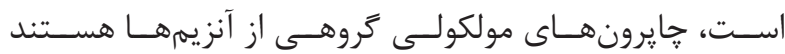

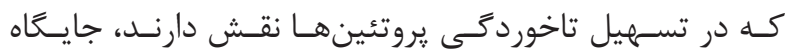

${ }^{26}$ Protein deglycase 1

${ }^{27}$ Dardarin

${ }^{28}$ Leucine rich repeat kinase 2

${ }^{29}$ Grb10-interacting GYFProtein2 


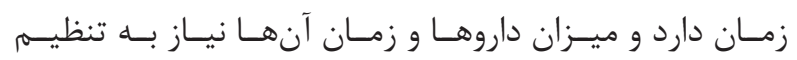

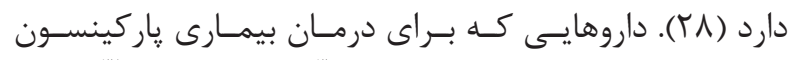

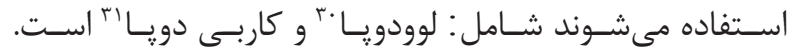

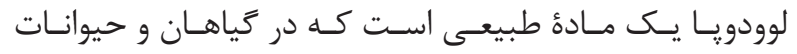

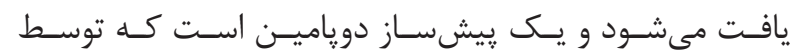

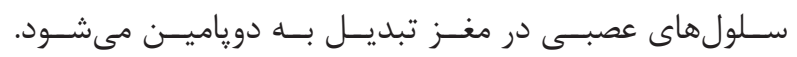

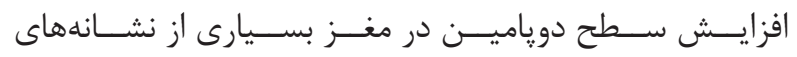

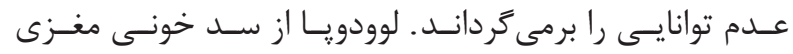

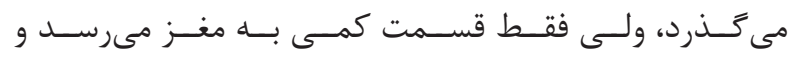

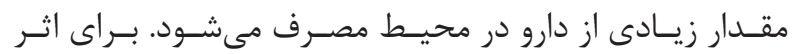

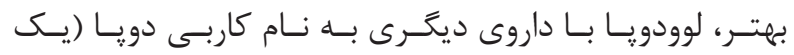

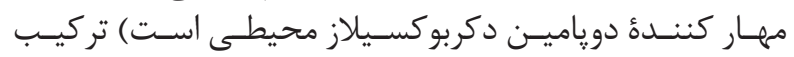

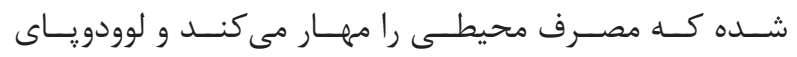

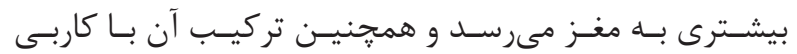

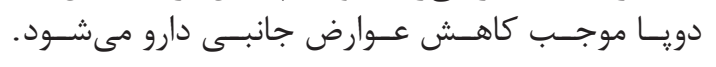

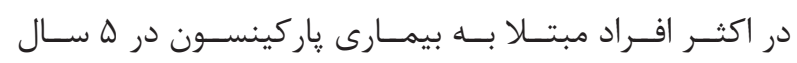

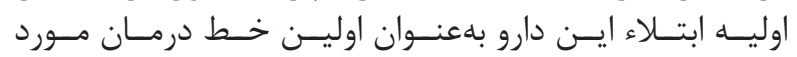

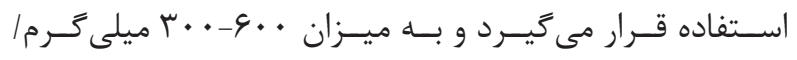

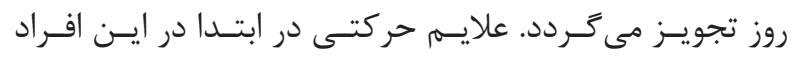

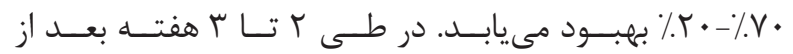

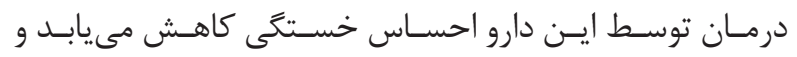

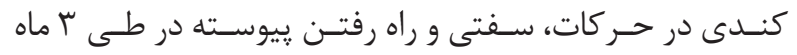

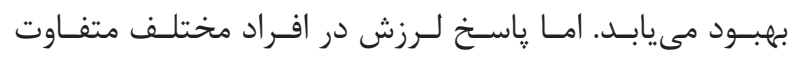

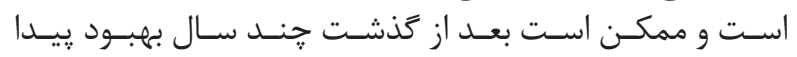

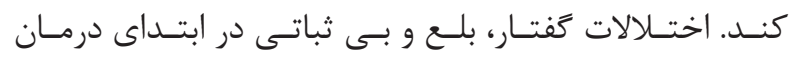

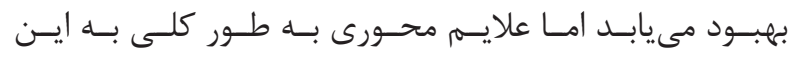

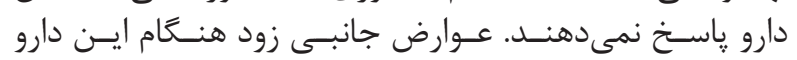

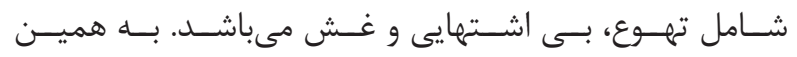

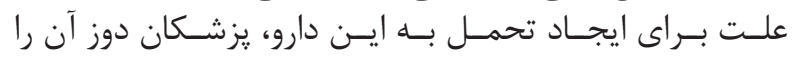

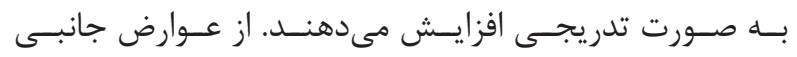

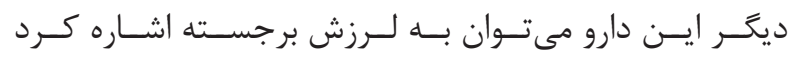

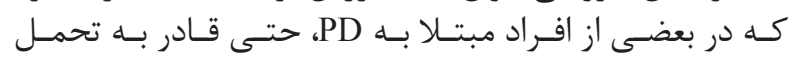

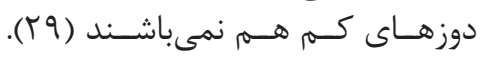

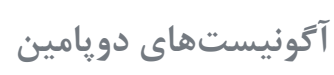

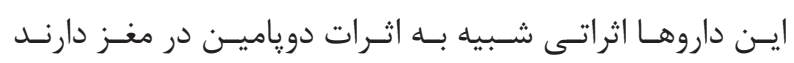

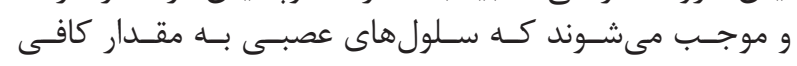

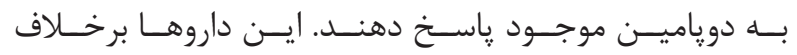

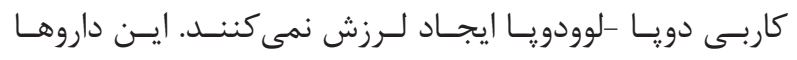

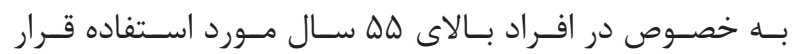

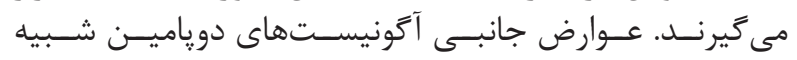

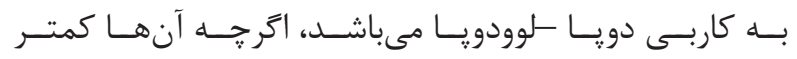

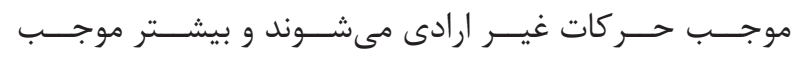

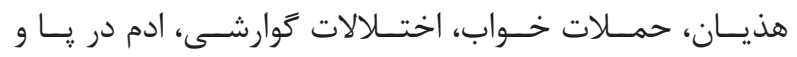

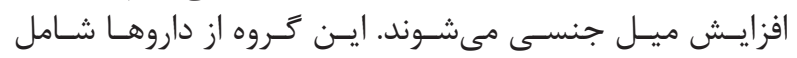

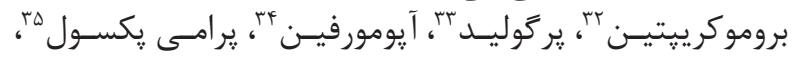

\footnotetext{
${ }^{30}$ Levodopa

${ }^{31}$ Carbidopa

${ }^{32}$ Bromocriptin
}

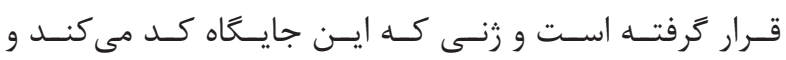

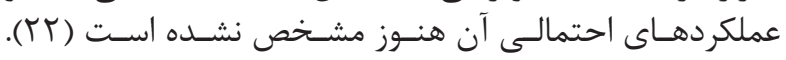

PARK13

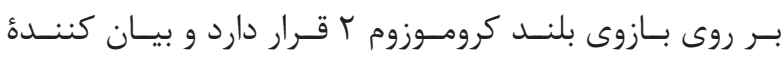

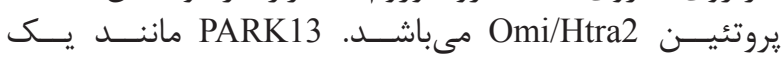

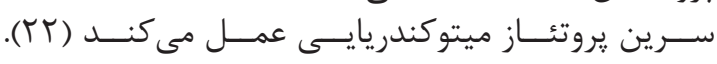

PARK14

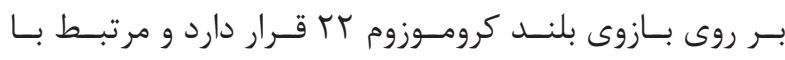

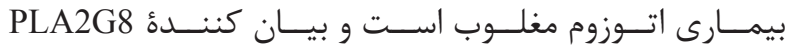

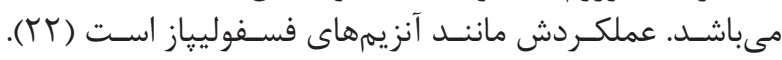

PARK15

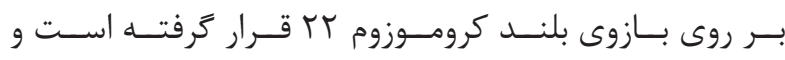

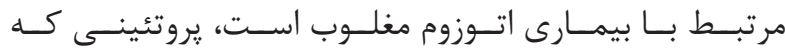

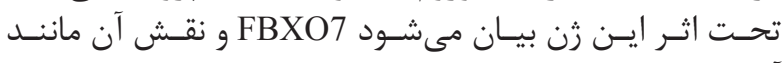

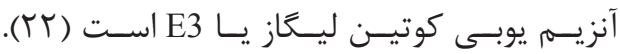

PARK16

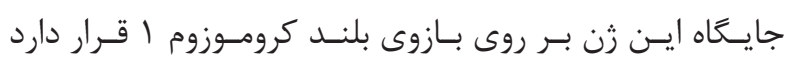

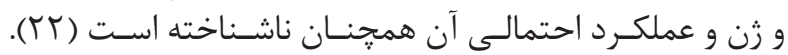

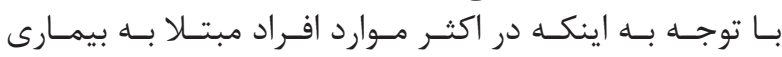

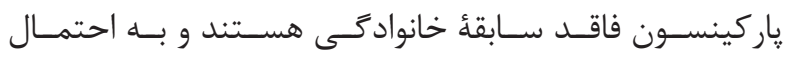

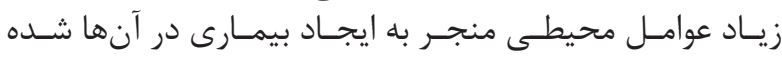

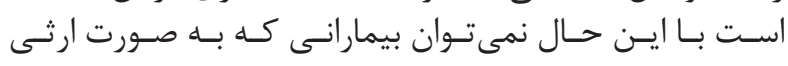

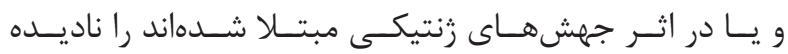

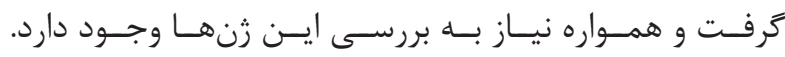

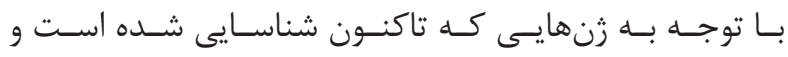

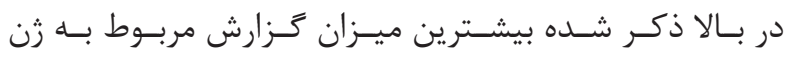
مA PARK2 و PARK1

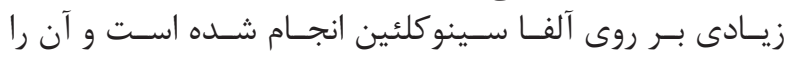

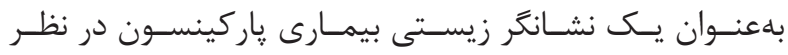

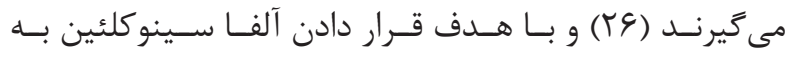

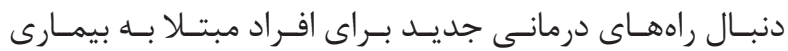

ياركينســون هسـتند (TV)

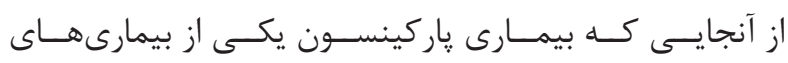

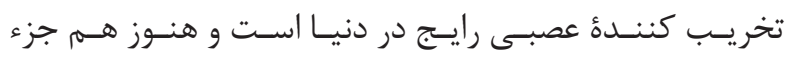

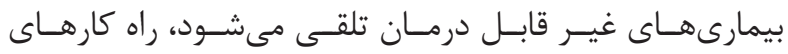

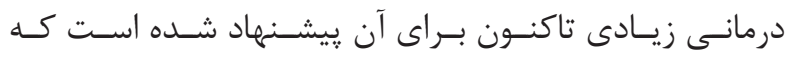

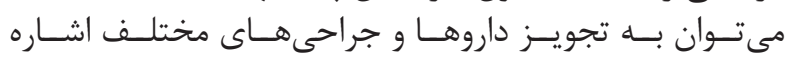

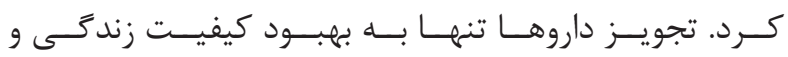

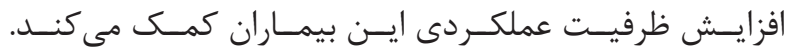

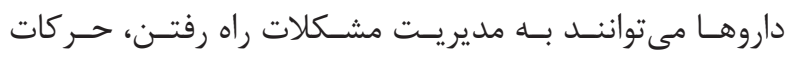

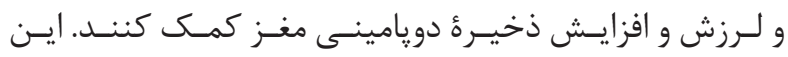

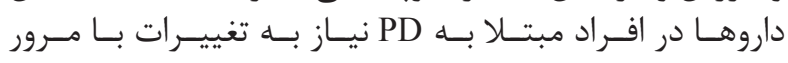

\footnotetext{
${ }^{33}$ Pergolid

${ }^{34}$ Apomorphin

${ }^{35}$ Pramipexole
} 
آمانتادين

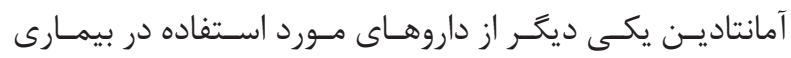

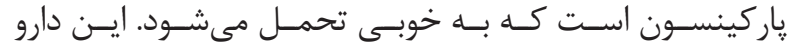

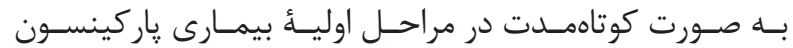

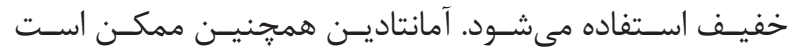

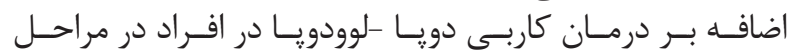

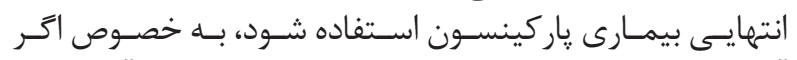

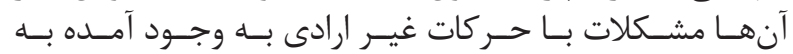

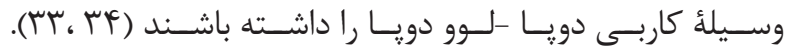

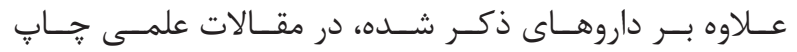

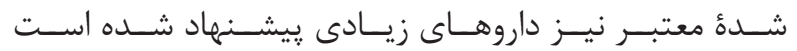

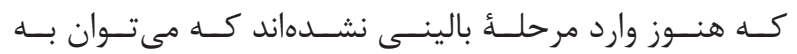

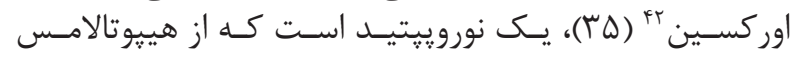

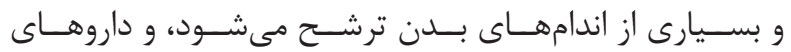

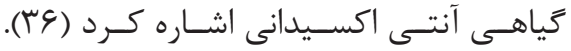

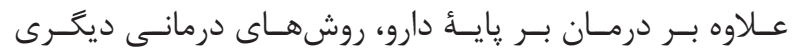

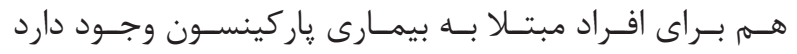

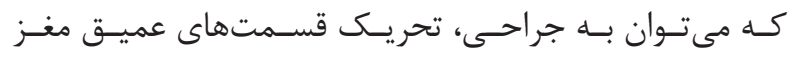

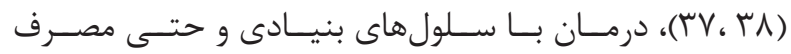

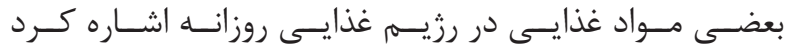

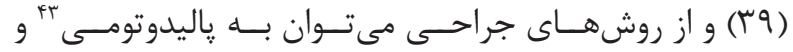

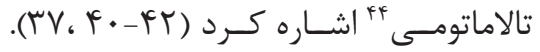

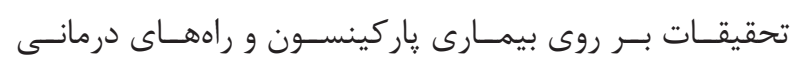

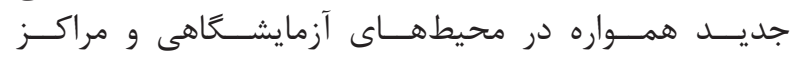

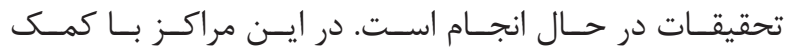

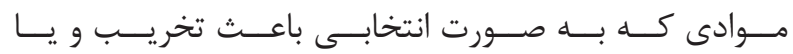

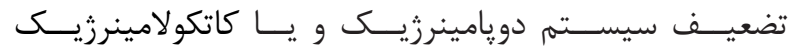

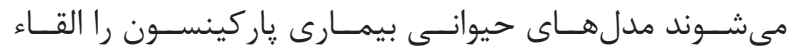

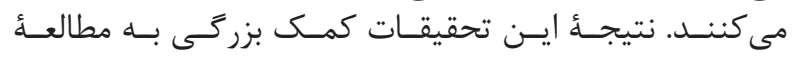

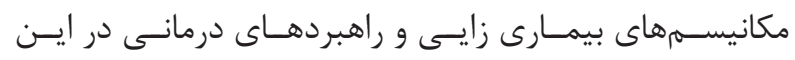

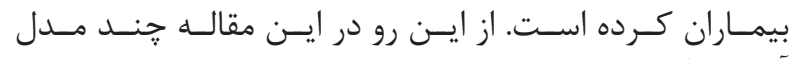

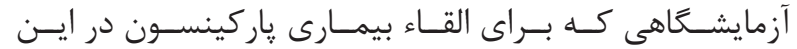

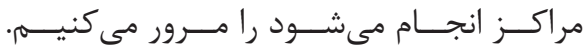

مدل رزريّن

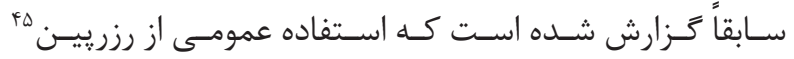

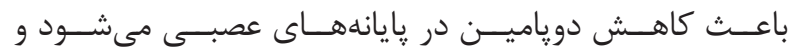

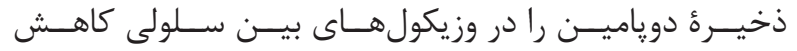

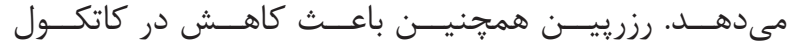

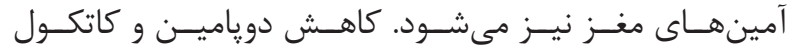

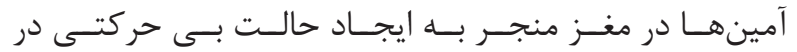

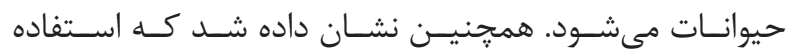

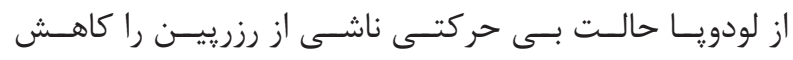

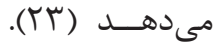

\footnotetext{
${ }^{36}$ Ropinirole

${ }^{37}$ Selegiline

${ }^{38}$ Rasageline

${ }^{39}$ Monoamine oxidase B

${ }^{40}$ Tolcapon
}

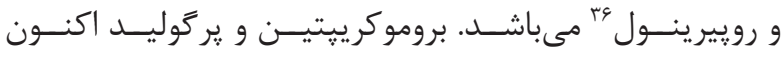

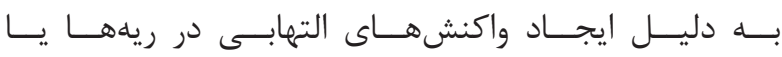

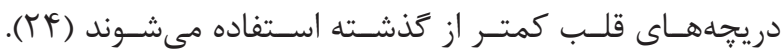

$$
\text { Bمهار كننده منوآمين اكسيداز }
$$

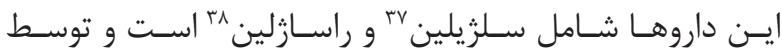

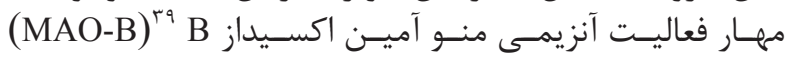

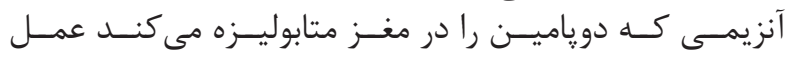

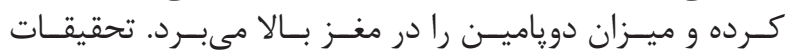

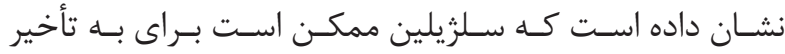

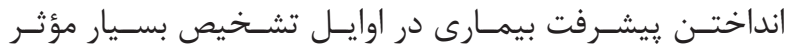

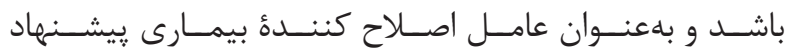

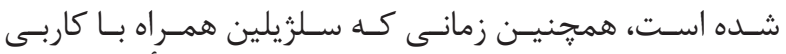

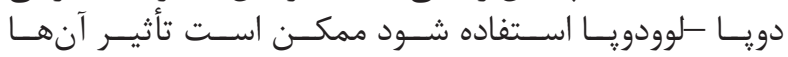

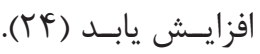

\section{COMT مهار كنندههاى}

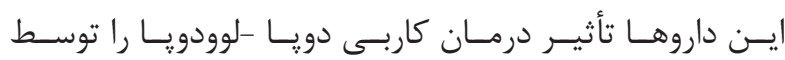

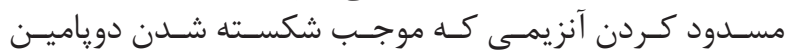

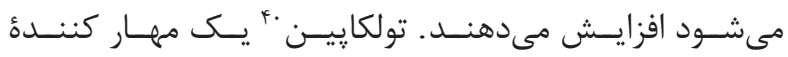

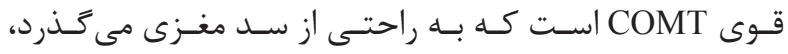

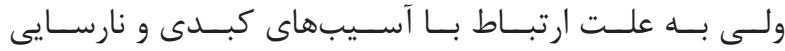

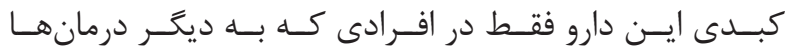

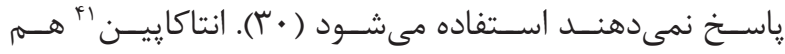

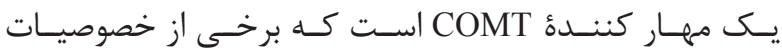

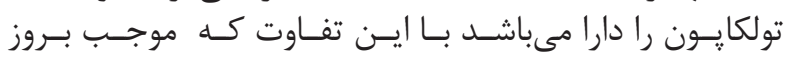

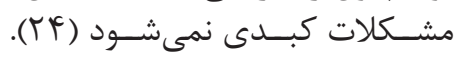

$$
\text { آنتى كولينرزيكها }
$$

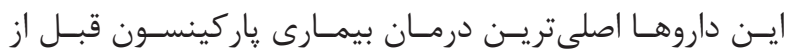

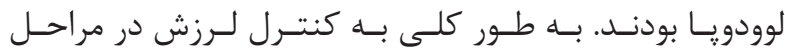

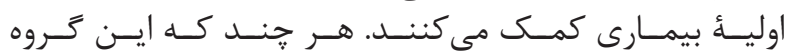

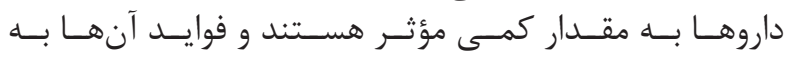

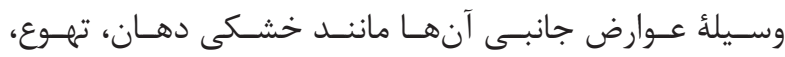

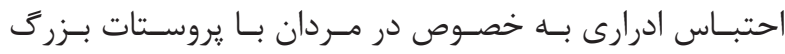

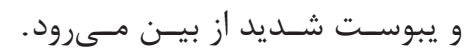

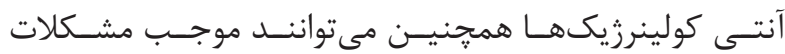

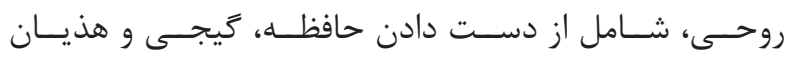

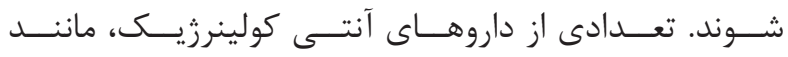

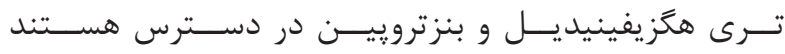

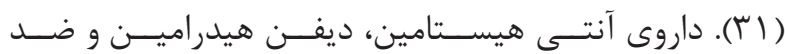

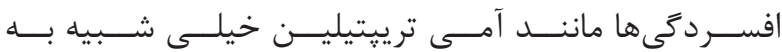

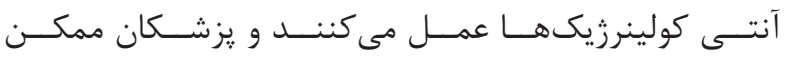

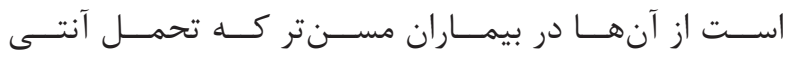

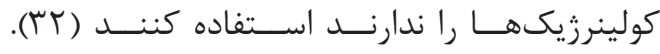

\footnotetext{
${ }^{41}$ Entacapone

${ }^{42}$ Orexin

${ }^{43}$ Pallidotomy

${ }^{44}$ Thalamatomy

${ }^{45}$ Reserpin
} 


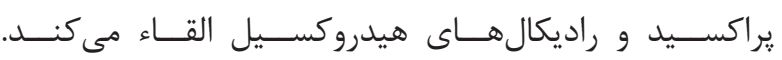

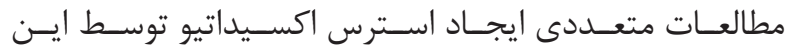

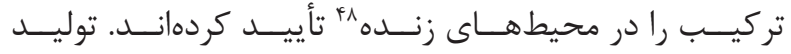

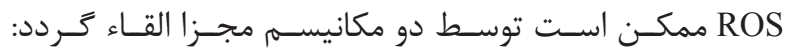

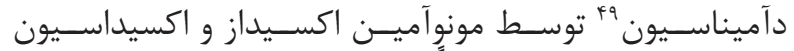

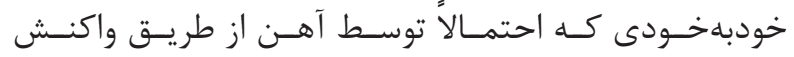

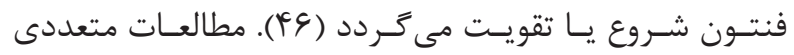

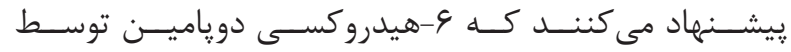

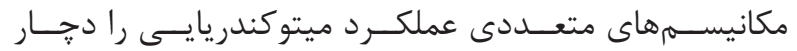

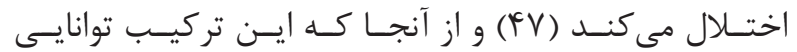

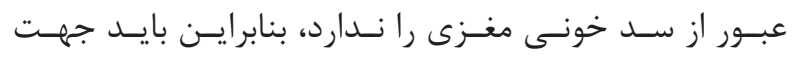

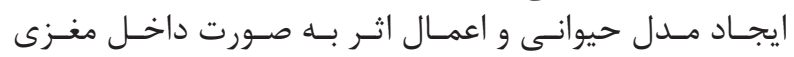

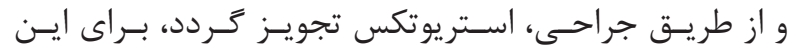

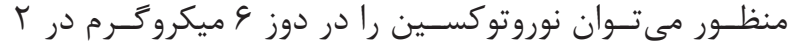

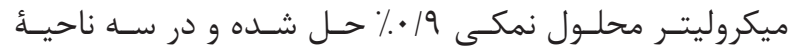

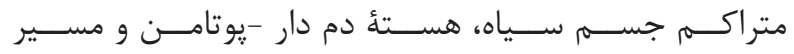

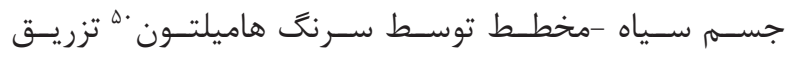

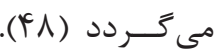

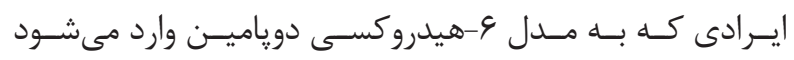

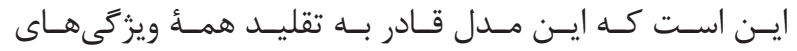

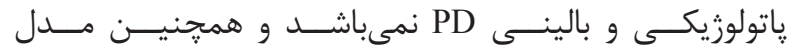

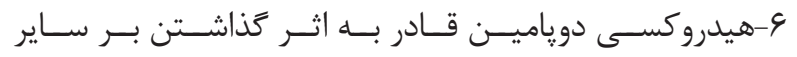

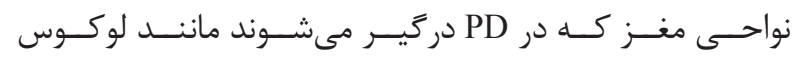

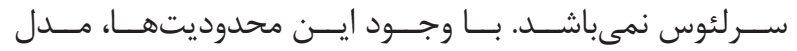

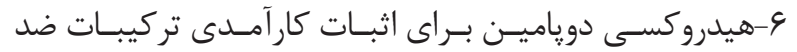

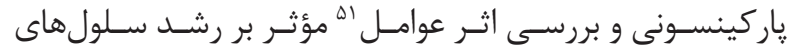

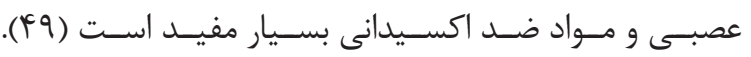

مدل MPTP

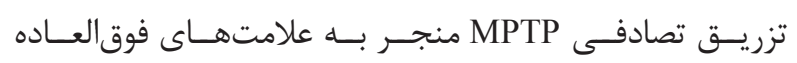

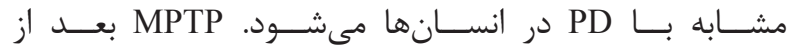

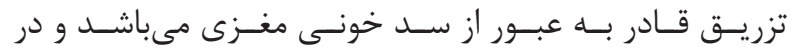

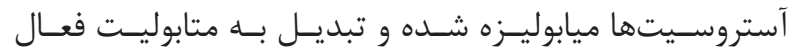

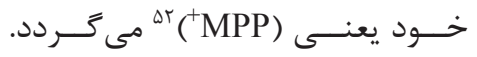

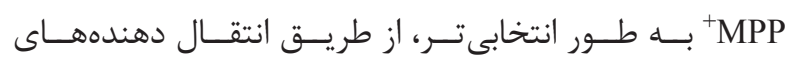

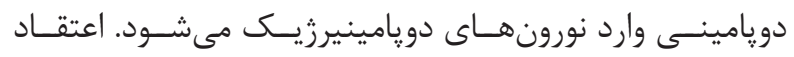

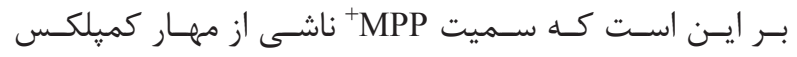

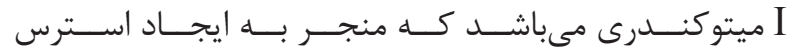

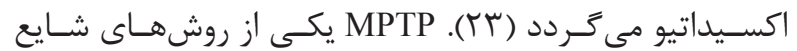

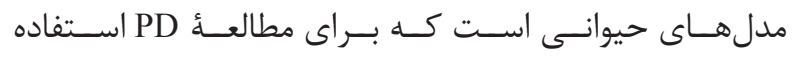

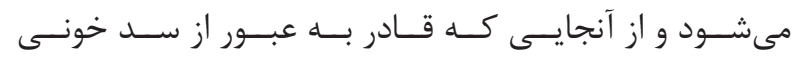

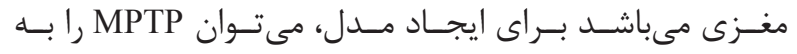

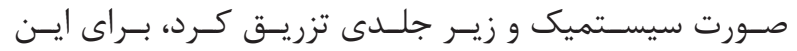

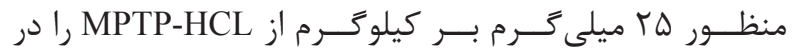

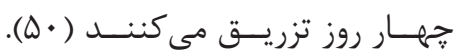

\footnotetext{
${ }^{46}$ Transporter

${ }^{47} 6$-hydroxydopamine

${ }^{48}$ In vivo

${ }^{49}$ Deamination
}

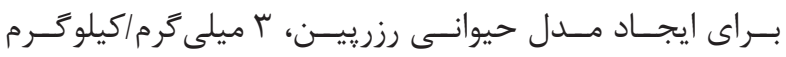

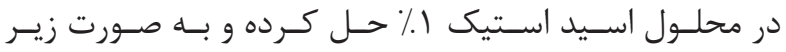

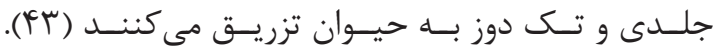

$$
\text { مدل مت آمفتامين }
$$

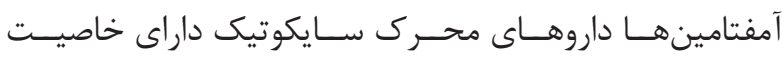

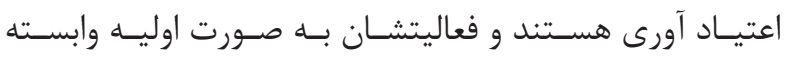

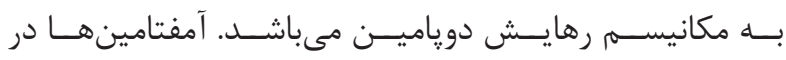

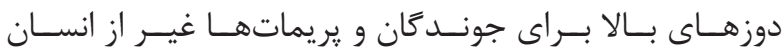

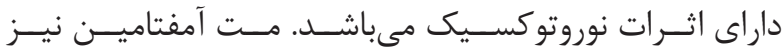

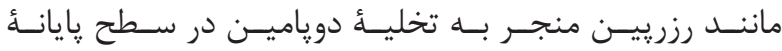

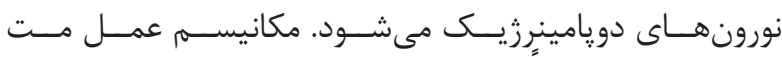

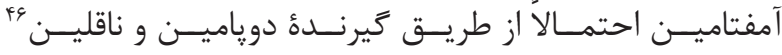

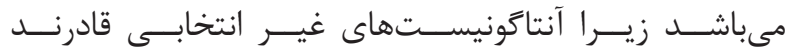

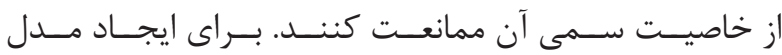

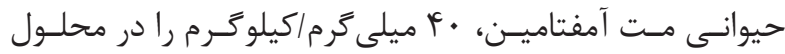

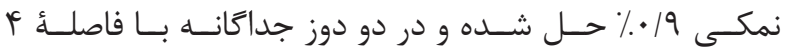

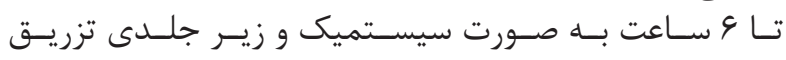

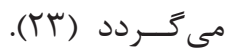

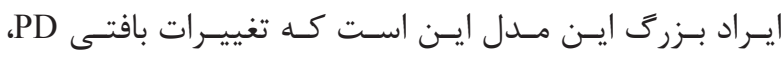

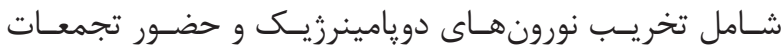

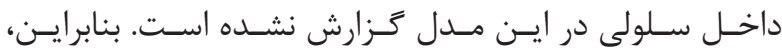

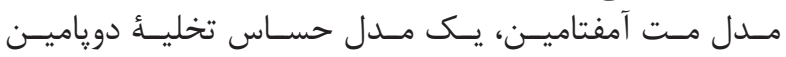

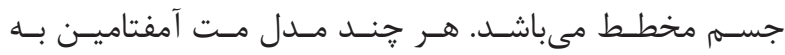

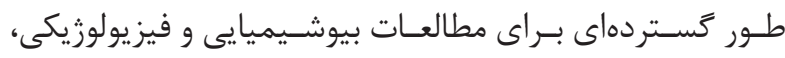

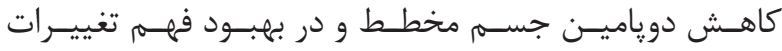

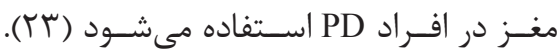

$$
\text { مدل و-هيدروكسى دويامين }
$$

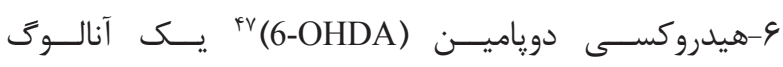

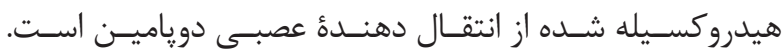

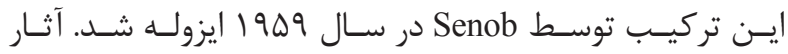

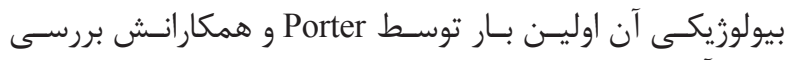

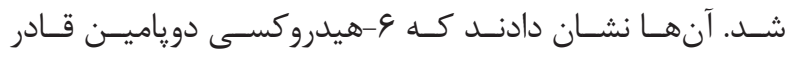

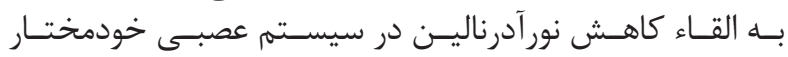

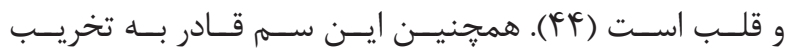

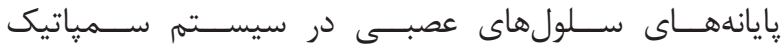

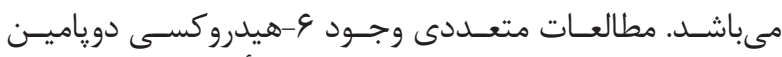

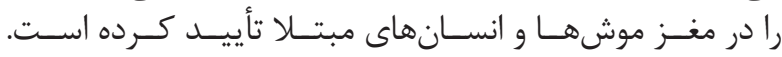

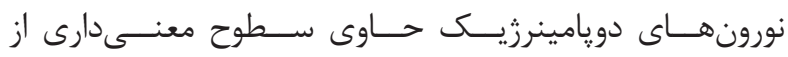

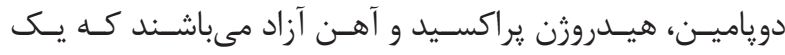

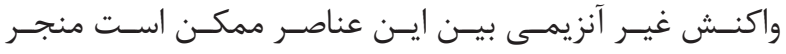

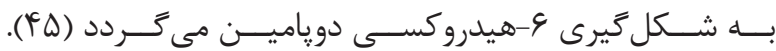

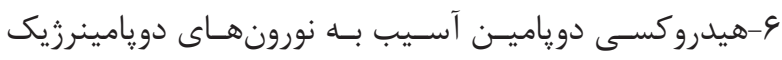

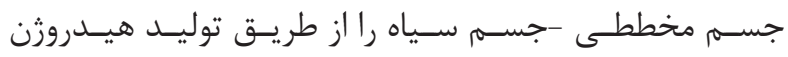

\footnotetext{
${ }^{50}$ Hamilton Syringe

${ }^{51}$ Factor

52 (1-methyl-4-phenylpyridinium
} 


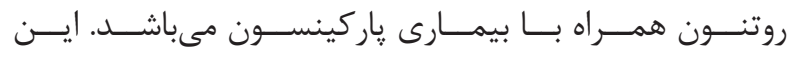

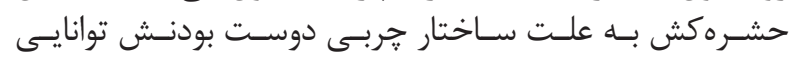

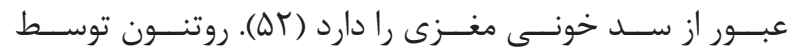

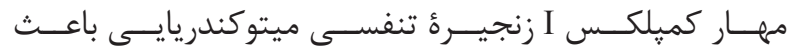

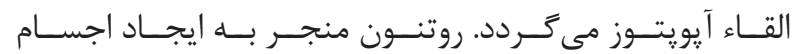

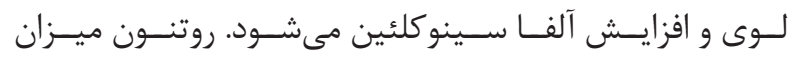

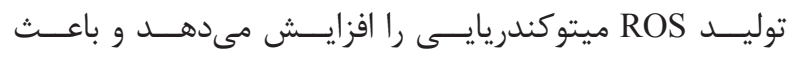

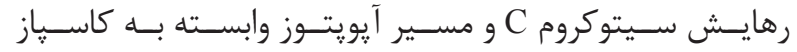

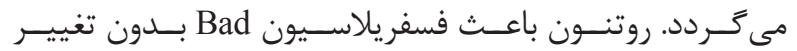

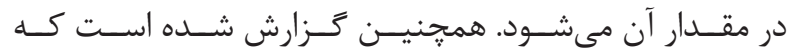

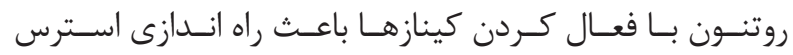

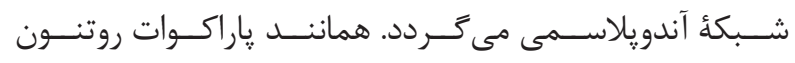

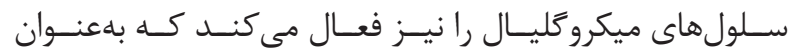

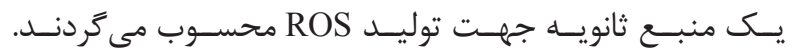

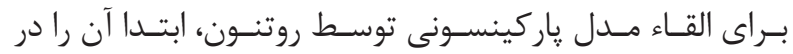

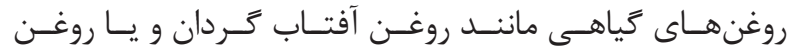

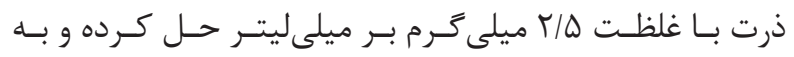

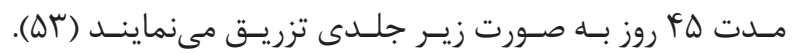

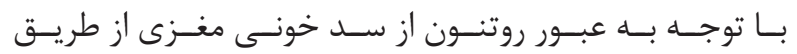

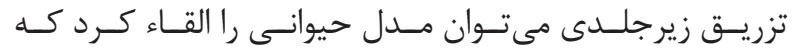

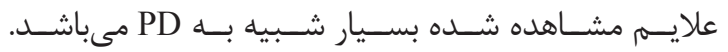
مدل هاى زُنتيكى مانى

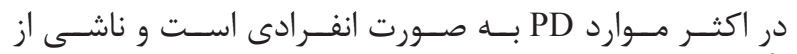

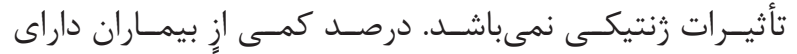

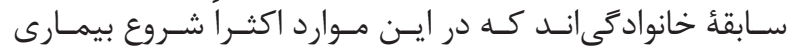

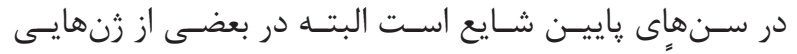

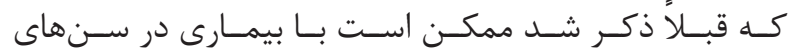

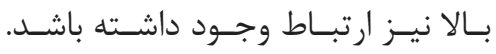

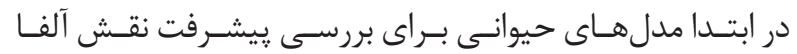

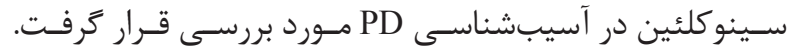

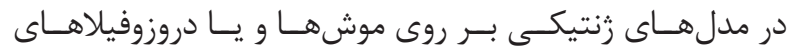

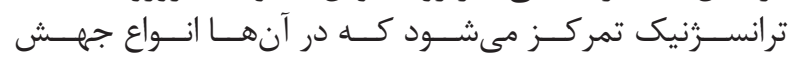

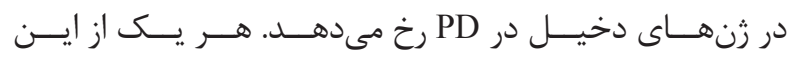

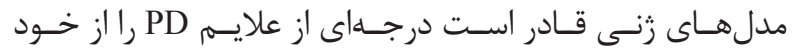

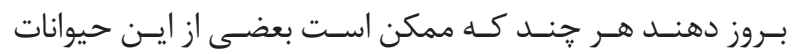

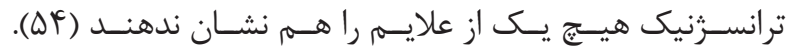

مدل كشت سلول

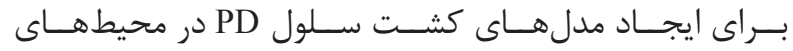

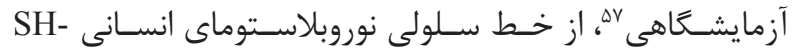

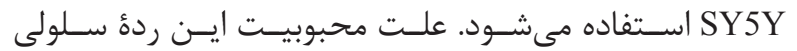

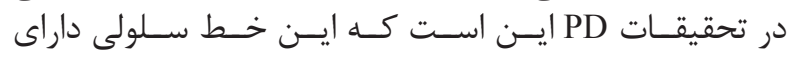

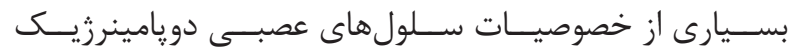

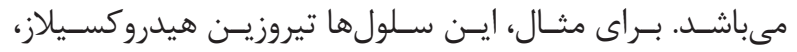

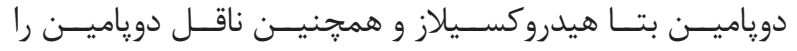

${ }^{53}$ 1,1'-Dimethyl-4,4'-bipyridinium dichloride

${ }^{54}$ Dopamine transporter

${ }^{55}$ Maneb

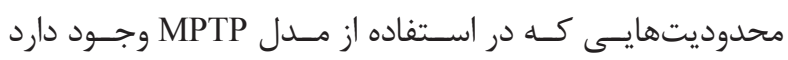

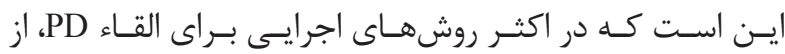

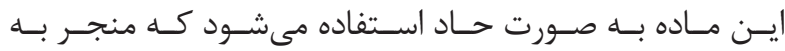

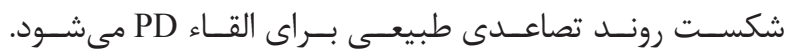

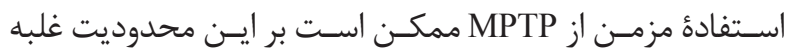

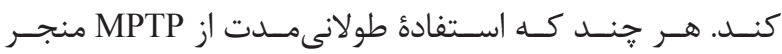

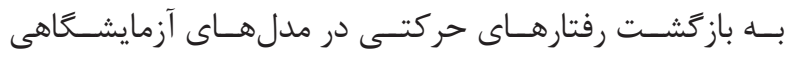

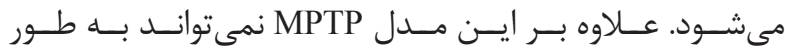

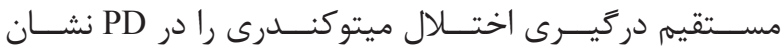

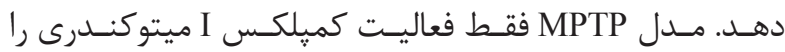

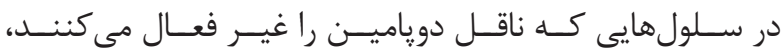

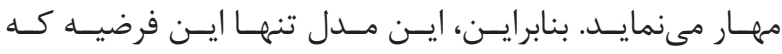

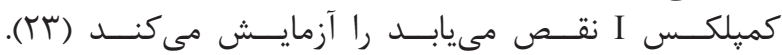
مدل هار اكوات

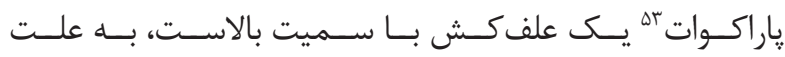

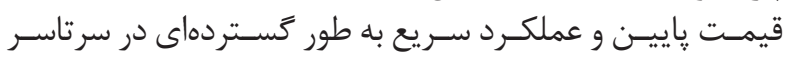

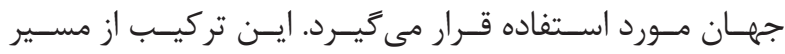

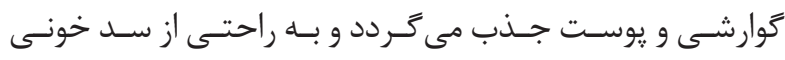

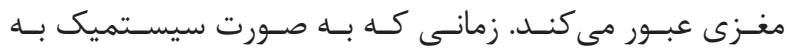

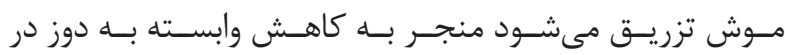

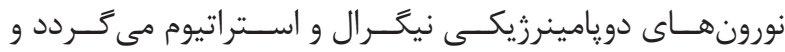

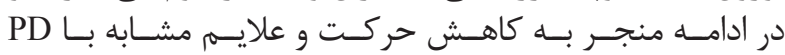

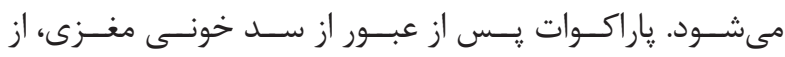

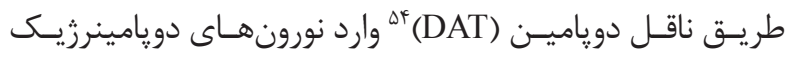

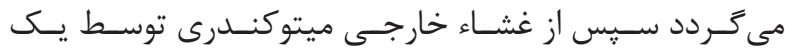

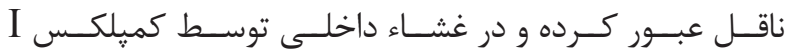

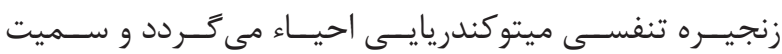

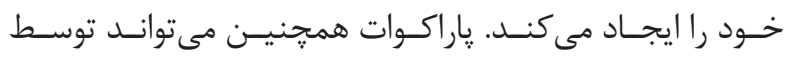

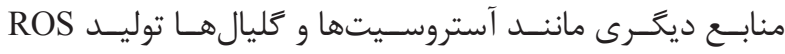

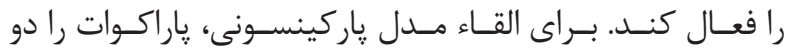

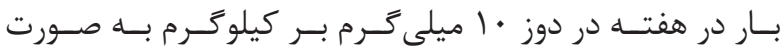

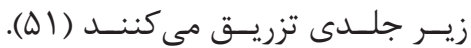
مدل مانب

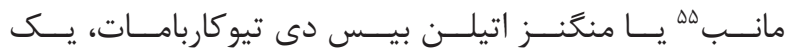

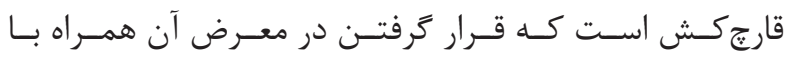

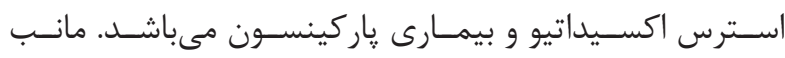

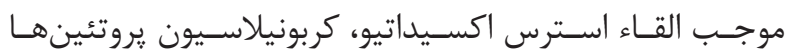

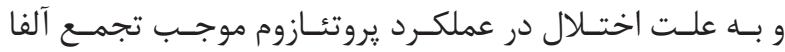

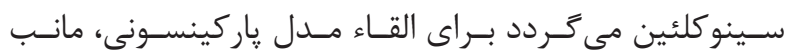

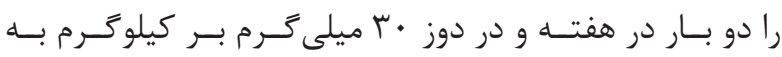

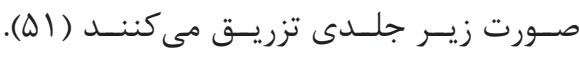
مدل مد روتنون

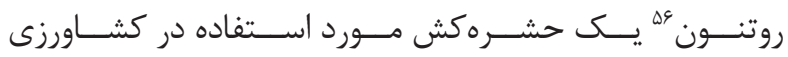

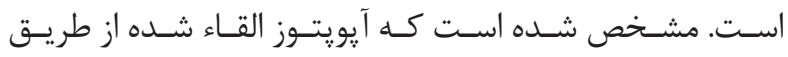

${ }^{56}$ Rotenone

${ }^{57}$ In vitro 


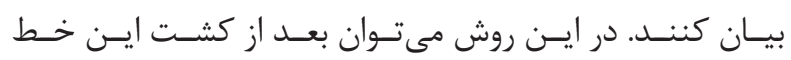

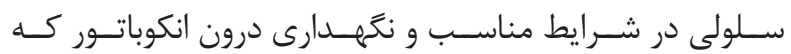

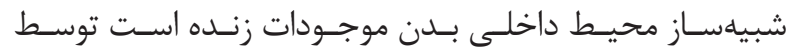

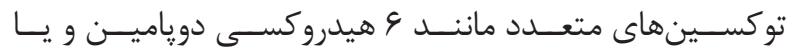

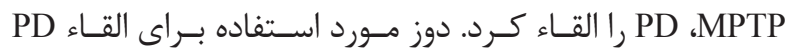

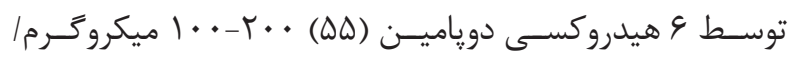

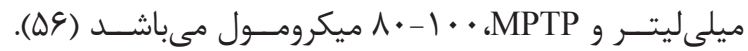
نتيجه

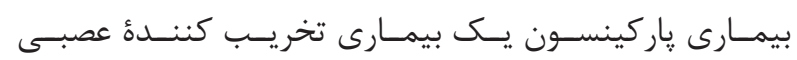

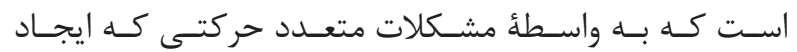

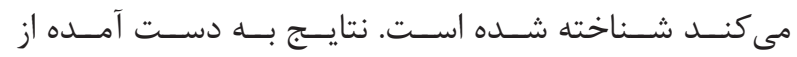

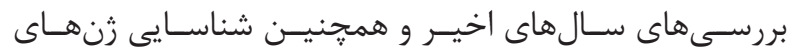

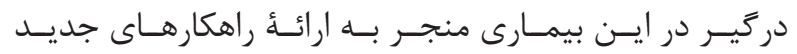

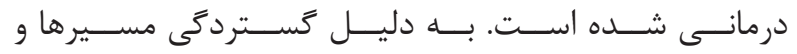

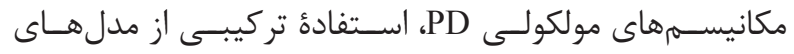

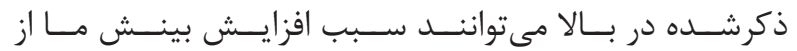

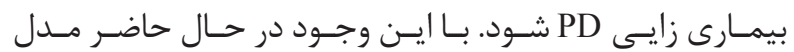

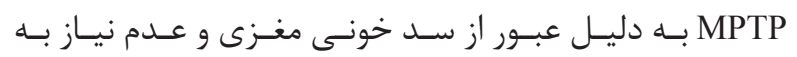

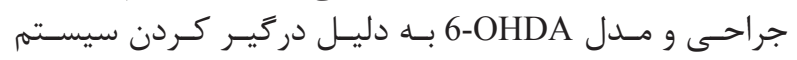

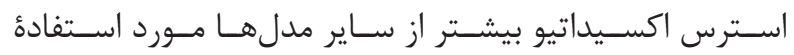
محققـان قــرار مى كيــرد.

1. Veldman BA, Wijn AM, Knoers N, Praamstra P, Horstink MW. Genetic and environmental risk factors in Parkinson's disease. J Clin Neurosci. 1998; 100(1): 15-26.

2. Spatola M, Wider C. Genetics of Parkinson's disease: the yield. Parkinsonism Relat Disord. 2014; 20(1): S35-S8.

3. Schapira AH. Neurobiology and treatment of Parkinson's disease. Trends Pharmacol Sci. 2009; 30(1): 41-7.

4. Elbaz A, Bower JH, Maraganore DM, McDonnell SK, Peterson BJ, Ahlskog JE, et al. Risk tables for parkinsonism and Parkinson's disease. J Clin Epidemiol. 2002; 55(1): 25-31.

5. Barrett KE, Barman SM, Boitano S. Ganong's review of medical physiology. $23^{\text {rd }}$ ed. New Yourk: McGraw Hill. 2010; p: 2010.

6. Savica R, Rocca WA, Ahlskog JE. When does Parkinson disease start? Archives of Neurology. 2010; 67(7): 798-801.

7. Boeve BF, Silber MH, Ferman TJ, Lucas JA,

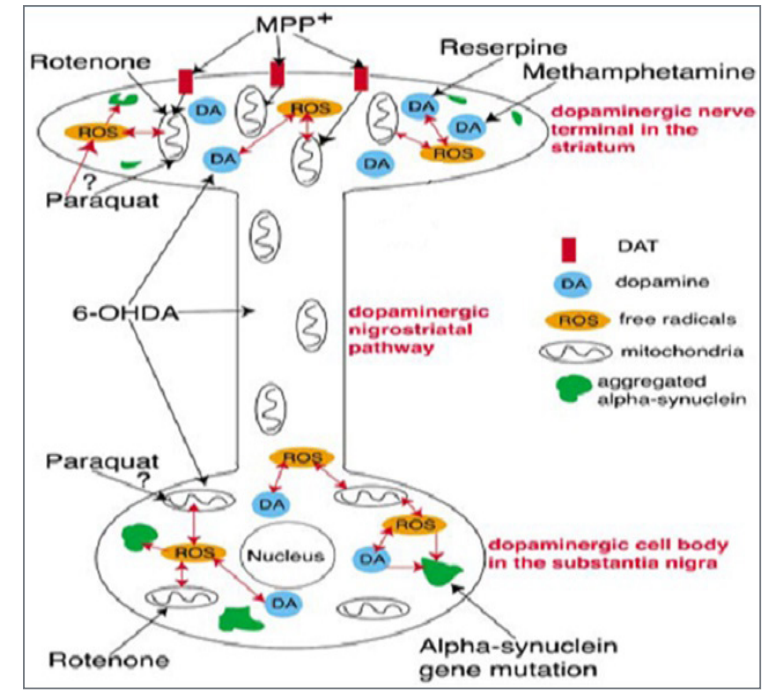

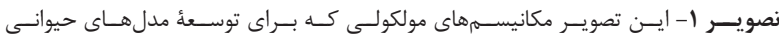

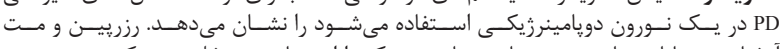

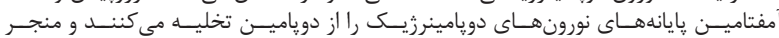

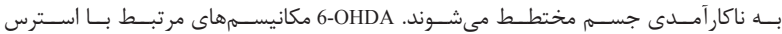

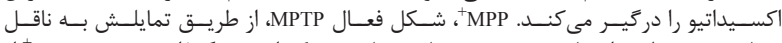

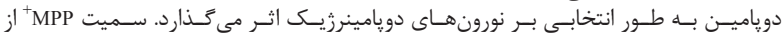

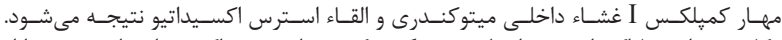

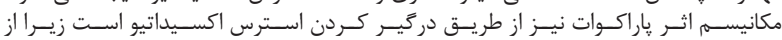

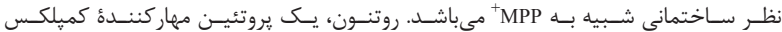

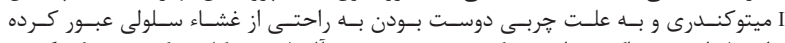

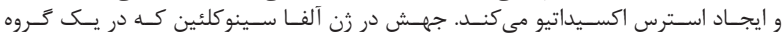

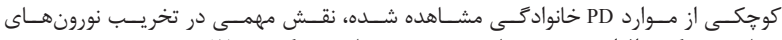

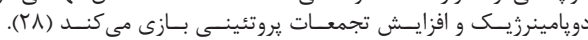

منابع

Parisi JE. Association of REM sleep behavior disorder and neurodegenerative disease may reflect an underlying synucleinopathy. Mov Disord. 2001; 16(4): $622-30$.

8. Lin T-K, Liou C-W, Chen S-D, Chuang Y-C, Tiao M-M, Wang P-W, et al. Mitochondrial dysfunction and biogenesis in the pathogenesis of Parkinson's disease. Chang Gung Med J. 2009; 32(6): 589-99.

9. Rascol O, Payoux P, Ory F, Ferreira JJ, BrefelCourbon C, Montastruc JL. Limitations of current Parkinson's disease therapy. Ann Neurol. 2003; 53(3): S12-5.

10. Berry C, La Vecchia C, Nicotera P. Paraquat and Parkinson's disease. Cell Death Differ. 2010; 17(7): 1115-25.

11. Wood-Kaczmar A, Gandhi S, Wood N. Understanding the molecular causes of Parkinson's disease. Trends Mol Med. 2006; 12(11): 521-8.

12. Franco R, Panayiotidis MI. Environmental toxicity, oxidative stress, human disease and the "black box" of their synergism: how much have we revealed? Mutat Res. 2009; 674(1-2): 1-2. 
13. Goedert M. Alpha-synuclein and neurodegenerative diseases. Nat Rev Neurosci. 2001; 2(7): 492-501.

14. Lotharius J, Brundin P. Pathogenesis of Parkinson's disease: dopamine, vesicles and $\alpha$-synuclein. Nat Rev Neurosci. 2002; 3(12): 932-42.

15. Hampe C, Ardila-Osorio H, Fournier M, Brice A, Corti O. Biochemical analysis of Parkinson's diseasecausing variants of Parkin, an E3 ubiquitin-protein ligase with monoubiquitylation capacity. Biochim Biophys Acta.. 2006; 15(13): 2059-75.

16. Levin L, Srour S, Gartner J, Kapitansky O, Qutob N, Dror $\mathrm{S}$, et al. Parkin somatic mutations link melanoma and Parkinson's disease. J Genet Genomics. 2016; 43(6): 369-79.

17. Yang Y, Gehrke S, Imai Y, Huang Z, Ouyang Y, Wang J-W, et al. Mitochondrial pathology and muscle and dopaminergic neuron degeneration caused by inactivation of drosophila pink1 is rescued by parkin. Proc Natl Acad Sci U S A. 2006; 103(28): 10793-8.

18. Pickrell AM, Youle RJ. The roles of pink1, parkin, and mitochondrial fidelity in Parkinson's disease. Neuron. 2015; 85(2): 257-73.

19. Foltynie T, Sawcer S, Brayne C, Barker R. The genetic basis of Parkinson's disease. J Neurol Neurosurg Psychiatry. 2002; 73(4): 363-70.

20. Ragland M, Hutter C, Zabetian C, Edwards K. Association between the ubiquitin carboxyl-terminal esterase L1 gene (UCHL1) S18Y variant and Parkinson's disease: a HuGE review and meta-analysis. Am J Epidemiol. 2009; 170(11): 1344-57.

21. Gandhi S, Muqit M, Stanyer L, Healy D, AbouSleiman P, Hargreaves I, et al. Pink1 protein in normal human brain and Parkinson's disease. Brain. 2006; 129(7): 1720-31.

22. Lockhart P, Lincoln S, Hulihan M, Kachergus J, Wilkes K, Bisceglio G, et al. DJ-1 mutations are a rare cause of recessively inherited early onset parkinsonism mediated by loss of protein function. J Med Genet. 2004; 41(3): e22. doi:10.1136/jmg.2003.011106.

23. Ferreira M, Massano J. An updated review of Parkinson's disease genetics and clinicopathological correlations. Acta Neurol Scand. 2016;1-12. doi: 10.1111/ane. 12616 .

24. Biskup S, West AB. Zeroing in on lrrk2-linked pathogenic mechanisms in Parkinson's disease. Biochim Biophys Acta. 2009; 1792(7): 625-33.
25. Thomas B, Beal MF. Molecular insights into Parkinson's disease. F1000 Med Rep. 2011; 3:7. doi:10.3410/M3-7.

26. Gao L, Tang H, Nie K, Wang L, Zhao J, Gan R, et al. Cerebrospinal fluid alpha-synuclein as a biomarker for Parkinson's disease diagnosis: a systematic review and meta-analysis. Int J Neurosci. 2015; 125(9): 64554.

27. Dehay B, Bourdenx M, Gorry P, Przedborski S, Vila M, Hunot S, et al. Targeting $\alpha$-synuclein for treatment of Parkinson's disease: mechanistic and therapeutic considerations. Lancet Neurol. 2015; 14(8): 855-66.

28. Betarbet R, Sherer TB, Greenamyre JT. Animal models of Parkinson's disease. Bio Essays. 2002; 24(4): 308-18.

29. Latoo J, Mistry M, Dunne FJ. Depression in Parkinson's disease: diagnosis and management. Br J Hosp Med. (17508460). 2012; 73(6): 331-4.

30. Müller T. Catechol-O-methyltransferase inhibitors in Parkinson's disease. Drugs. 2015; 75(2): 157-74.

31. Ehrt U, Broich K, Larsen JP, Ballard C, Aarsland D. Use of drugs with anticholinergic effect and impact on cognition in Parkinson's disease: a cohort study. J Neurol Neurosurgery Psychiatry. 2010; 81(2): 160-5.

32. Simola N, Pinna A, Fenu S. Pharmacological therapy of Parkinson's disease: current options and new avenues. Recent Pat CNS Drug Discov. 2010; 5(3): 221-38.

33. Onofrj M, Frazzini V, Bonanni L, Thomas A. Amantadine and antiglutamatergic drugs in the management of Parkinson's disease. Parkinson's Disease: Current and Future Therapeutics and Clinical Trials. $1^{\text {st }}$ ed.Cambridge University Press. 2016.

34. Silva J, Monge-Fuentes V, Gomes F, Lopes K, Anjos Ld, Campos G, et al. Pharmacological alternatives for the treatment of neurodegenerative disorders: wasp and bee venoms and their components as new neuroactive tools. Toxins. 2015; 7(8): 3179-209.

35. Esmaeili-Mahani S, Vazifekhah S, Pasban-Aliabadi H, Abbasnejad M, Sheibani V. Protective effect of orexin-A on 6-hydroxydopamine-induced neurotoxicity in SH-SY5Y human dopaminergic neuroblastoma cells. Neurochem Int. 2013; 63(8): 719-25.

36. Solesio ME, Prime TA, Logan A, Murphy MP, del Mar Arroyo-Jimenez M, Jordán J, et al. The mitochondria-targeted anti-oxidant MitoQ reduces aspects of mitochondrial fission in the 6-OHDA cell 


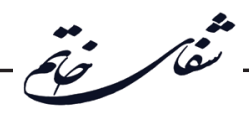

model of Parkinson's disease. Biochim Biophys Acta. 2013; 1832(1): 174-82.

37. Tarsy D, Vitek JL, Lozano AM. Surgical treatment of Parkinson's disease and other movement disorders. Humana Press, Totowa (NJ); 2003:189-212.

38. Politis M, Lindvall O. Clinical application of stem cell therapy in Parkinson's disease. BMC Medicine. 2012; 10(1): 1. doi: 10.1186/1741-7015-10-1

39. Aligholi H, Safahani M, Sarkaki A, Amani R. Protective effect of soy on movement disorders induced by parkinson disease in ovariectomized animal model. Shefaye Khatam. 2013; 1(3): 5-10.

40. Gross RE, Lozano AM, Lang AE, Tasker RR, Hutchison WD, Dostrovsky JO. The effects of pallidotomy on Parkinson's disease: study design and assessment techniques. Acta Neurochir. 1997; 68: 24-8.

41. Elias WJ, Huss D, Voss T, Loomba J, Khaled M, Zadicario E, et al. A pilot study of focused ultrasound thalamotomy for essential tremor. N Engl J Med. 2013; 369(7): 640-8.

42 Ahmadi M, Sharifi M S. Treatments of Parkinson's disease, epilepsy and obsessive compulsive disorder with deep brain stimulation. Shefaye Khatam. 2014; 2 (1) :95-100.

43. Nash JE, Hill MP, Brotchie JM. Antiparkinsonian actions of blockade of NR2B-containing NMDA receptors in the reserpine-treated rat. Exp Neurol. 1999; 155(1): 42-8.

44. Thoenen H, Tranzer J. Chemical sympathectomy by selective destruction of adrenergic nerve endings with 6-hydroxydopamine. Naunyn Schmiedebergs Arch Pharmakol. 1968; 261(3): 271-88.

45. Hefti F, Melamed E, Sahakian BJ, Wurtman RJ. Circling behavior in rats with partial, unilateral nigrostriatal lesions: effect of amphetamine, apomorphine, and DOPA. Pharmacol Biochem Behav 1980; 12(2): $185-8$.

46. Blum D, Torch S, Lambeng N, Nissou M-F, Benabid A-L, Sadoul R, et al. Molecular pathways involved in the neurotoxicity of 6-OHDA, dopamine and MPTP: contribution to the apoptotic theory in Parkinson's disease. Prog Neurobiol. 2001; 65(2): 135-72.

47. Storch A, Kaftan A, Burkhardt K, Schwarz J. 6-Hydroxydopamine toxicity towards human $\mathrm{SH}-$ SY5Y dopaminergic neuroblastoma cells: independent of mitochondrial energy metabolism. J Neural Transm. 2000; 107(3): 281-93.

48. Blandini F, Armentero M-T, Martignoni E. The 6-hydroxydopamine model: news from the past. Parkinsonism Relat Disord. 2008; 14: S124-S9.

49. Jin B, Iacovitti L. Dopamine differentiation factors produce partial motor recovery in 6-hydroxydopamine lesioned rats. Neurobiol Dis. 1995; 2(1): 1-12.

50. Hantraye P, Brouillet E, Ferrante R, Palfi S, Dolan $\mathrm{R}$, Matthews RT, et al. Inhibition of neuronal nitric oxide synthase prevents MPTP-induced parkinsonism in baboons. Nat Med. 1996; 2(9): 1017-21.

51. Singhal NK, Srivastava G, Patel DK, Jain SK, Singh MP. Melatonin or silymarin reduces maneb-and paraquat-induced Parkinson's disease phenotype in the mouse. J Pineal Res. 2011; 50(2): 97-109.

52. Alam M, Schmidt W. Rotenone destroys dopaminergic neurons and induces parkinsonian symptoms in rats. Behav Brain Res. 2002; 136(1): 317-24.

53. Klintworth H, Newhouse K, Li T, Choi W-S, Faigle $\mathrm{R}, \mathrm{Xia} \mathrm{Z}$. Activation of c-Jun $\mathrm{N}$-terminal protein kinase is a common mechanism underlying paraquat-and rotenone-induced dopaminergic cell apoptosis. Toxicol Sci.. 2007; 97(1): 149-62.

54. Dawson TM, Ko HS, Dawson VL. Genetic animal models of Parkinson's disease. Neuron. 2010; 66(5): 646-61.

55. Guo S, Bezard E, Zhao B. Protective effect of green tea polyphenols on the SH-SY5Y cells against 6-OHDA induced apoptosis through ROS-NO pathway. Free Radic Biol Med. 2005; 39(5): 682-95.

56. Sheehan JP, Palmer PE, Helm GA, Tuttle JB. MPP+ induced apoptotic cell death in SH-SY5Y neuroblastoma cells: an electron microscope study. J Neurosci Res. 1997; 48(3): 226-37. 